\title{
A capped trigonal pyramidal molybdenum hydrido complex and an unusually mild sulfur- carbon bond cleavage reaction
}

\author{
Robert H. Morris \\ Version Post-print/accepted manuscript \\ Citation R. H. Morris, Chemical Communications, 2017, 53, 11032 - \\ (published version) 11035. \\ http://doi.org/10.1039/C7CC06372E
}

Copyright / License

Publisher's Statement This document is the Accepted Manuscript version of a Published Work that appeared in final form in Chemical Communications, copyright $\odot$ Royal Society of Chemistry after peer review and technical editing by the publisher. To access the final edited and published work see http://doi.org/10.1039/C7CC06372E.

How to cite TSpace items

Always cite the published version, so the author(s) will receive recognition through services that track citation counts, e.g. Scopus. If you need to cite the page number of the author manuscript from TSpace because you cannot access the published version, then cite the TSpace version in addition to the published version using the permanent URI (handle) found on the record page.

This article was made openly accessible by $U$ of $T$ Faculty. Please tell us how this access benefits you. Your story matters. 


\section{Accepted Manuscript}

This article can be cited before page numbers have been issued, to do this please use: R. H. Morris, Chem. Commun., 2017, DOI: 10.1039/C7CC06372E.
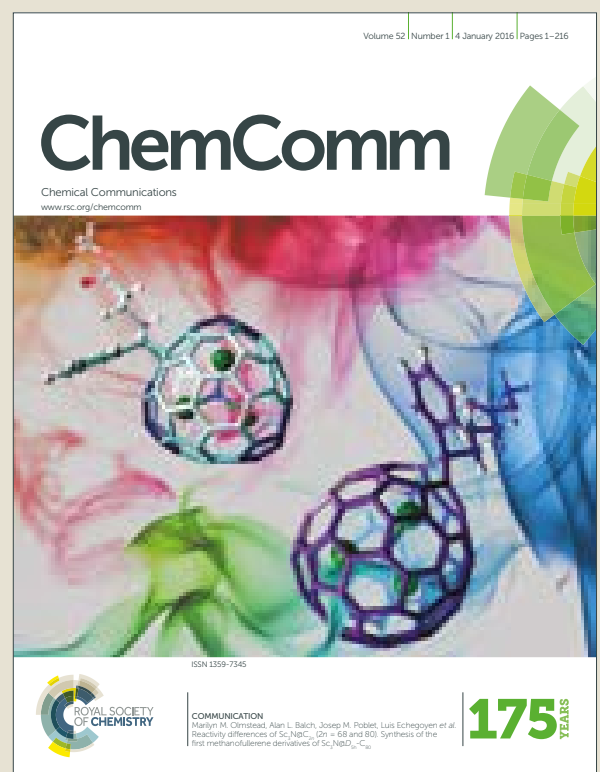

This is an Accepted Manuscript, which has been through the Royal Society of Chemistry peer review process and has been accepted for publication.

Accepted Manuscripts are published online shortly after acceptance, before technical editing, formatting and proof reading. Using this free service, authors can make their results available to the community, in citable form, before we publish the edited article. We will replace this Accepted Manuscript with the edited and formatted Advance Article as soon as it is available.

You can find more information about Accepted Manuscripts in the author guidelines.

Please note that technical editing may introduce minor changes to the text and/or graphics, which may alter content. The journal's standard Terms \& Conditions and the ethical guidelines, outlined in our author and reviewer resource centre, still apply. In no event shall the Royal Society of Chemistry be held responsible for any errors or omissions in this Accepted Manuscript or any consequences arising from the use of any information it contains. 


\section{Journal Name}

\section{COMMUNICATION}

\section{A capped trigonal pyramidal molybdenum hydrido complex and an unusually mild sulfur-carbon bond cleavage reaction}

Received 00th January 20xx, Accepted 00th January 20xx

DOI: $10.1039 / \mathrm{x} 0 \times x \times 00000 \mathrm{x}$

www.rsc.org/

DFT calculations reveal that a reported molybdenum hydride complex has an unprecedented geometry with the molybdenum in the base of trigonal pyramid defined by three thiolate ligands with a phosphine ligand on the apex and a hydride capping a $\mathrm{PS}_{2}$ face. This complex reacts with methanol to produce a sulfido complex by a new reaction: the protonation of an ipso carbon of a thiolate ligand by coordinated methanol.

Hydrodesulfurization (HDS) is an important step in the upgrading of petroleum to clean fuels, usually using catalysts based on molybdenum disulfide. ${ }^{1} \quad$ There is theoretical evidence for the formation of unstable molybdenum hydrides $(\mathrm{MoH})$ and molybdenum hydrosulfide (MoSH) groups on the surface of the heterogeneous catalyst. ${ }^{2,3}$ The MoSH groups are thought to be more stable under HDS conditions. ${ }^{3}$ The mechanism of carbon-sulfur bond cleavage is still under investigation. Inorganic chemists are studying the reactions of well-defined metal complexes to establish the mechanisms of carbon-sulfur bond cleavage. ${ }^{4}$ For example, low valent molybdenum complexes have been shown to cleave $\mathrm{C}-\mathrm{H}$ and $\mathrm{S}-$ C bonds. ${ }^{5}$ The mechanisms of $\mathrm{H}_{2}$ activation at sulfido-bridged dimolybdenum cyclopentadienyl complexes have been investigated computationally and found to occur either homolytically between sulfur ligands ${ }^{6 a}$ or heterolytically across a Mo-S bond ${ }^{6 \mathrm{~b}}$ depending on the complex.

Some of us were involved in the study of homogenous, diamagnetic molybdenum hydridoarylthiolato complexes $\mathrm{MoH}(\mathrm{SAr})_{3}\left(\mathrm{PRPh}_{2}\right), \mathrm{R}=\mathrm{Me}, \mathrm{Et}, \mathrm{Ar}=2,4,6-\mathrm{PPr}_{3} \mathrm{C}_{6} \mathrm{H}_{2}$ and 2,4,6$\mathrm{Me}_{3} \mathrm{C}_{6} \mathrm{H}_{2}$ that underwent a spontaneous $\mathrm{S}-\mathrm{C}$ cleavage event at a sulfur-aryl bond when their THF solutions were exposed to methanol. ${ }^{7,} 8$ When deuterated methanol $\left(\mathrm{MeO}^{2} \mathrm{H}\right)$ was employed, monodeuterated arene was detected along with non-deuterated free thiol and a dimeric molybdenum complex

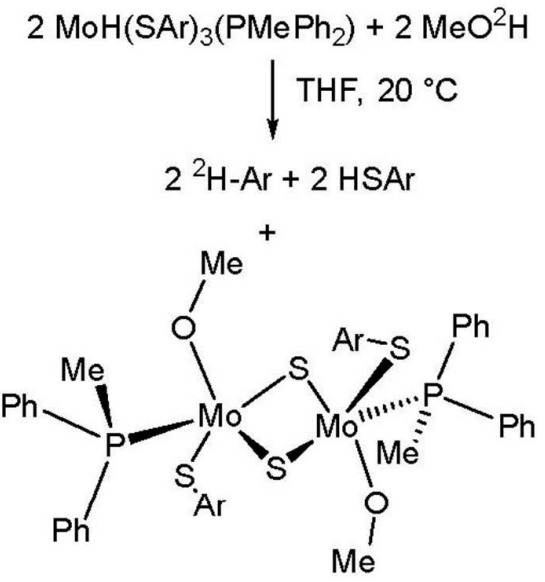

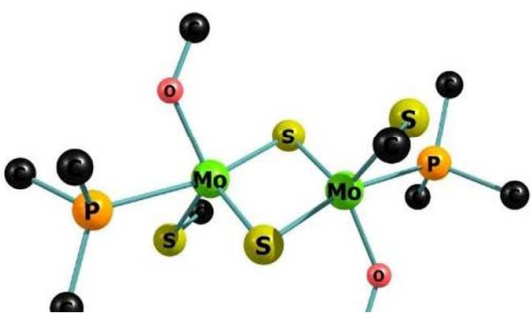

Figure 1. The cleavage of sulfur-carbon bonds under mild conditions by reaction of $\mathrm{MeO}^{2} \mathrm{H}$ with $\mathrm{MoH}\left(\mathrm{PMePh}_{2}\right)(\mathrm{SAr})_{3}, \mathrm{Ar}=2,4,6-\mathrm{Me}_{3} \mathrm{C}_{6} \mathrm{H}_{2}$. The structure of the dimer was determined by single crystal X-ray diffraction ${ }^{7,8}$ and a similar $\mathrm{PMe}_{3}$ dimer $\left(\mathrm{Mo}_{2}\right)$ by DFT calculation (this work). The hydrogens and all carbons except those attached to the donor atoms have been removed for clarity. The level of theory for this calculation and the others shown in this article is M11-L with basis set SDD on Mo and 6-31G(d) on the other atoms with THF solvation applied using the PCM model unless otherwise specified.

$\left(\mathrm{Mo}_{2}\right)$ with two bridging sulfido ligands (Figure 1). ${ }^{8}$ The $\mathrm{Mo}_{2}$ product was characterized by single crystal X-ray diffraction but we were unable to obtain suitable crystals of the starting hydride complex.

\footnotetext{
Department of Chemistry, University of Toronto, 80 Saint George St., Toronto Ontario, M5S3H6, Canada.E-mail: rmorris@chem.utoronto.ca

+ Electronic Supplementary Information (ESI) available: Computational details, coordinates and energies of the structures. See DOI: 10.1039/x0xx00000x
} 


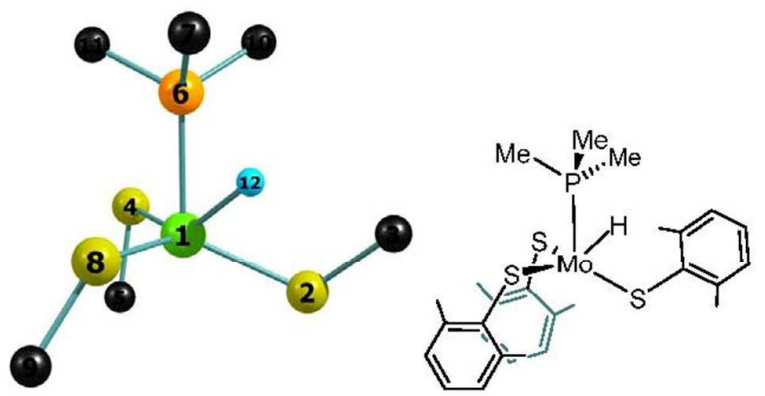

Figure 2. The structure of $\mathrm{MoH}\left(\mathrm{PMe}_{3}\right)\left(\mathrm{SAr}_{3}\right.$ with all of the hydrogens on the ligands and all of the carbons apart from the ipso carbons removed. Selected bond distances $(\AA ̊)$ and angles $\left({ }^{\circ}\right)$ : $\mathrm{Mo}(1)-\mathrm{H}(12) 1.65, \mathrm{Mo}(1)-\mathrm{P}(6)$ 2.37, $\mathrm{Mo}(1)-\mathrm{S}(2)$ 2.34, $\mathrm{Mo}(1)-\mathrm{S}(4) 2.36$, $\mathrm{Mo}(1)-\mathrm{S}(8)$ 2.33; H(12)-Mo(1)-P(6) 54.1, H(12)-Mo(1)-S(2) 73.5, H(12)-Mo(1)-S(8) 84.6, $\mathrm{P}(6)-\mathrm{Mo}(1)-\mathrm{S}(2)$ 115.0, P(6)-Mo(1)-S(4) 84.6, P(6)-Mo(1)-S(8) 91.3, S(2)-Mo(1)-S(4) 112.9, S(4)-Mo(1)-S(8) 121.1, S(8)-Mo(1)-S(2) 121.6.

The molybdenum in $\mathrm{MoH}(\mathrm{SAr})_{3}\left(\mathrm{PRPh}_{2}\right)$ was proposed to be in a trigonal bipyramidal geometry with the hydride trans to the phosphine ligand on the basis of the observation in the NMR spectra of a large ${ }^{2} J_{\text {HP }}$ coupling constant ranging from 79 to $87 \mathrm{~Hz}$ between the hydride, detected at $3.3 \mathrm{ppm}$ for one of the compounds, and the phosphorus nuclei. ${ }^{7,8}$ However DFT calculations using a slightly simplified structure $\left(\mathrm{Mo}\left(\mathrm{PMe}_{3}\right)(\mathrm{H})(\mathrm{SAr})_{3}, \mathrm{Ar}=2,6-\mathrm{Me}_{2} \mathrm{C}_{6} \mathrm{H}_{3}\right)$ now provide strong evidence that the most stable isomer of these complexes has molybdenum in an unprecedented distorted capped trigonal pyramidal (CTP) geometry (Figure 2 ).

In this diamagnetic, five coordinate complex the molybdenum sits $0.3 \AA$ above the middle of a distorted triangle defined by the three sulfur atoms (Figure 2). The phosphorus is at the vertex of the distorted $S_{3} P$ trigonal pyramid with the short Mo-P bond $\left(2.37 \AA\right.$ ) at a $78^{\circ}$ angle to the $S_{3}$ basal plane. The hydride ligand caps one $\mathrm{PS}_{2}$ face of this CTP with a small HMoP angle of $54.1^{\circ}$. Thus the large ${ }^{2} J_{H P}$ is associated with this small angle between these nuclei. There are no distortions of the $\mathrm{PMe}_{3}$ ligand that would indicate bonding favouring 5coordinate phosphorus. The HOMO is a lone pair with approximately $\left(d_{x z}\right)^{2}(\mathrm{Mo})$ character, out of phase with a $\left(p_{z}\right)^{2}$ lone pair on $\mathrm{S} 8$ (see the $\mathrm{SI}$ ) where the $\mathrm{x}$ direction is along the $\mathrm{Mo}-\mathrm{S} 8$ bond and the $\mathrm{z}$ direction is between the Mo-H and Mo$P$ bonds. None of the 620 five-coordinate complexes containing a terminal hydride found in the Cambridge Structural Database has such a CTP geometry. Two isomers with the hydride approximately trans to the phosphorus in a distorted trigonal bipyramidal (TBP) geometry were located as stable minima at 10 and $13 \mathrm{kcal} / \mathrm{mol}$ higher in energy and a distorted trigonal bipyramidal complex in the triplet state was located at $5 \mathrm{kcal} / \mathrm{mol}$ higher in energy. Even the model complex $\mathrm{Mo}\left(\mathrm{PMe}_{3}\right)(\mathrm{H})(\mathrm{SMe})_{3}$ with much smaller substituents on the sulfurs adopts this CTP geometry. Apparently molybdenum is large enough to accommodate ligands cis to each other, thus avoiding trans H-Mo-P and H-Mo-S bond weakening. The ${ }^{2} J_{H P}$ (unscaled) were calculated using the gauge-independent atomic orbital method as $-70 \mathrm{~Hz}$ for the CTP geometry and $-31 \mathrm{~Hz}$ for the TBP geometry. The former

2 | J. Name., 2012, 00, 1-3 value agrees better with the range from experiment (79 to 87 $\mathrm{Hz}, \pm$ sign not determined).

This CTP geometry explains why the complex $\mathrm{MoH}\left(\mathrm{PMePh}_{2}\right)(\mathrm{SAr})_{3}, \mathrm{Ar}=2,4,6-\mathrm{iPr}_{3} \mathrm{C}_{6} \mathrm{H}_{2}$ readily reacts with pyridine to give the complex $\mathrm{MoH}\left(\mathrm{PMePh}_{2}\right)(\mathrm{py})(\mathrm{SAr})_{3}, \mathrm{Ar}=$ 2,4,6-i $\mathrm{Pr}_{3} \mathrm{C}_{6} \mathrm{H}_{2}$, now known to have a capped trigonal bipyramidal (CTB) geometry ${ }^{9}$ with the pyridine across from the hydride and the phosphorus at a small angle to the hydride, producing $\mathrm{a}^{2} J_{\mathrm{HP}}$ of $87 \mathrm{~Hz}$ (eq 1 ).

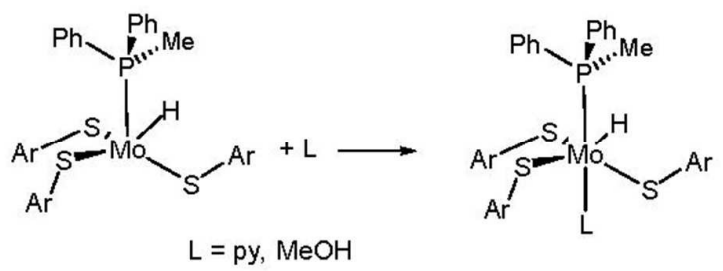

The original proposal for the sulfur-carbon bond cleavage of Figure 1 was that the hydride would attack the carbon; ${ }^{8}$ however this would involve isotope exchange with the hydride

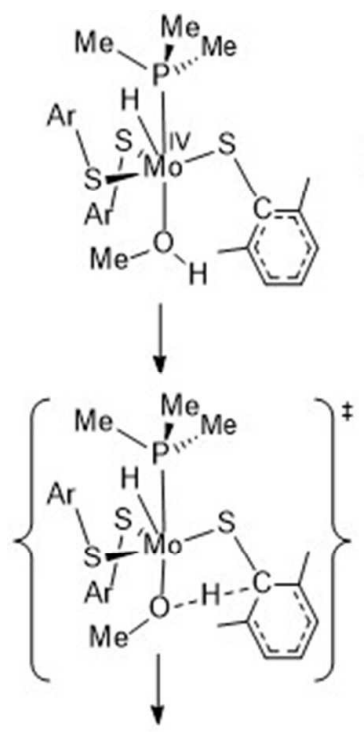

(a)

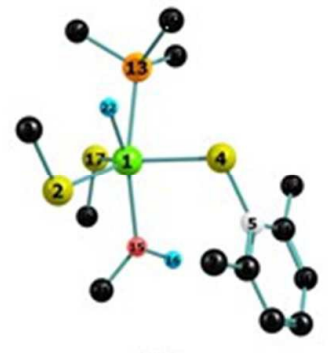

(b)

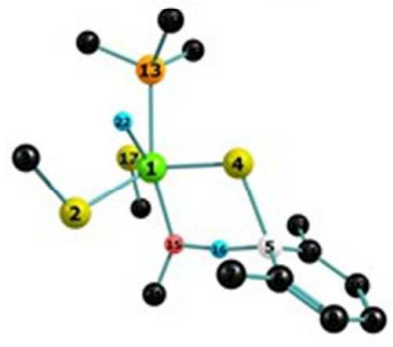

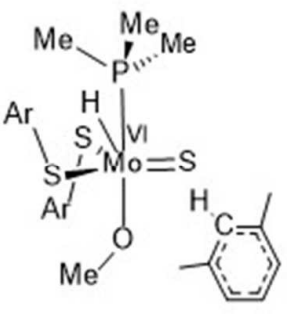

(c)

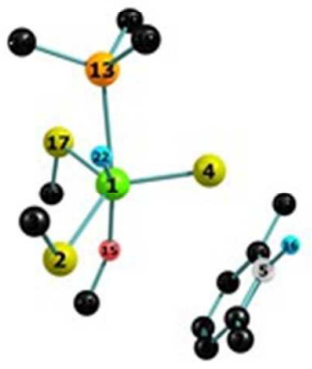

that was not observed and our DFT studies could not find a suitable transition state for the hydride attack. The methanol that is needed to trigger this cleavage reaction (Figure 1) can easily coordinate to give a formally Mo(IV) methanol complex as shown in eq 1 . From this experimentally unobserved methanol adduct, the $\mathrm{C}-\mathrm{S}$ bond is readily cleaved in an

Figure 3. (a) The methanol adduct $\mathrm{MoH}\left(\mathrm{PR}_{3}\right)(\mathrm{OHMe})(\mathrm{SAr})_{3}(\mathrm{Mo}(1)-\mathrm{H}(22) 1.66, \mathrm{Mo}(1)$ $\mathrm{P}(13)$ 2.39, $\mathrm{Mo}(1)-\mathrm{O}(15)$ 2.21, $\mathrm{Mo}(1)-\mathrm{S}(2)$ 2.36, $\mathrm{Mo}(1)-\mathrm{S}(4)$ 2.33, $\mathrm{Mo}(1)-\mathrm{S}(17) 2.36$, $\mathrm{O}(15)-\mathrm{H}(16)$ 0.97, S(4)-C(5) $1.78 \AA ̊$; H(22)-Mo(1)-P(13) 52.1, H(22)-Mo(1)-O(15) 148.5, $\left.\mathrm{P}(13)-\mathrm{Mo}(1)-\mathrm{O}(15) 158.7, \mathrm{Mo}(1)-\mathrm{S}(4)-\mathrm{C}(5) 113.0^{\circ}\right)$; (b) the hydrogen transfer transition state (OH...CSMo) (1635i cm ${ }^{-1}$; Mo(1)-H(22) 1.66, Mo(1)-P(13) 2.45, Mo(1)-O(15) 2.07, $\mathrm{Mo}(1)-\mathrm{S}(2)$ 2.37, $\mathrm{Mo}(1)-\mathrm{S}(4)$ 2.20, $\mathrm{Mo}(1)-\mathrm{S}(17)$ 2.38, O(15)-H(16) 1.21, S(4)-C(5) 2.14, $\mathrm{H}(16)-\mathrm{C}(5) 1.33 \AA ̊$; $\mathrm{H}(22)-\mathrm{Mo}(1)-\mathrm{P}(13)$ 54.3, H(22)-Mo(1)-O(15) 146.7, $\mathrm{P}(13)-\mathrm{Mo}(1)-\mathrm{O}(15)$ 157.7, $\left.\mathrm{Mo}(1)-\mathrm{S}(4)-\mathrm{C}(5) 107.1^{\circ}\right)$; (c) the sulfido product $\mathrm{MoH}(\mathrm{S})\left(\mathrm{PR}_{3}\right)(\mathrm{OMe})(\mathrm{SAr})_{2} \cdot \mathrm{HAr}$ (Mo(1)-H(22) 1.65, Mo(1)-P(13) 2.56, Mo(1)-O(15) 1.93, Mo(1)-S(2) 2.40, Mo(1)-S(4) 2.14, Mo(1)-S(17) $2.42 \AA ̊$; H(22)-Mo(1)-P(13) 54.8, H(22)-Mo(1)-O(15) 145.6, P(13)Mo(1)-O(15) $155.7^{\circ}$ plus $\mathrm{HAr}(\mathrm{C}(5)-\mathrm{H}(16) 1.10 \AA ̊)$. 


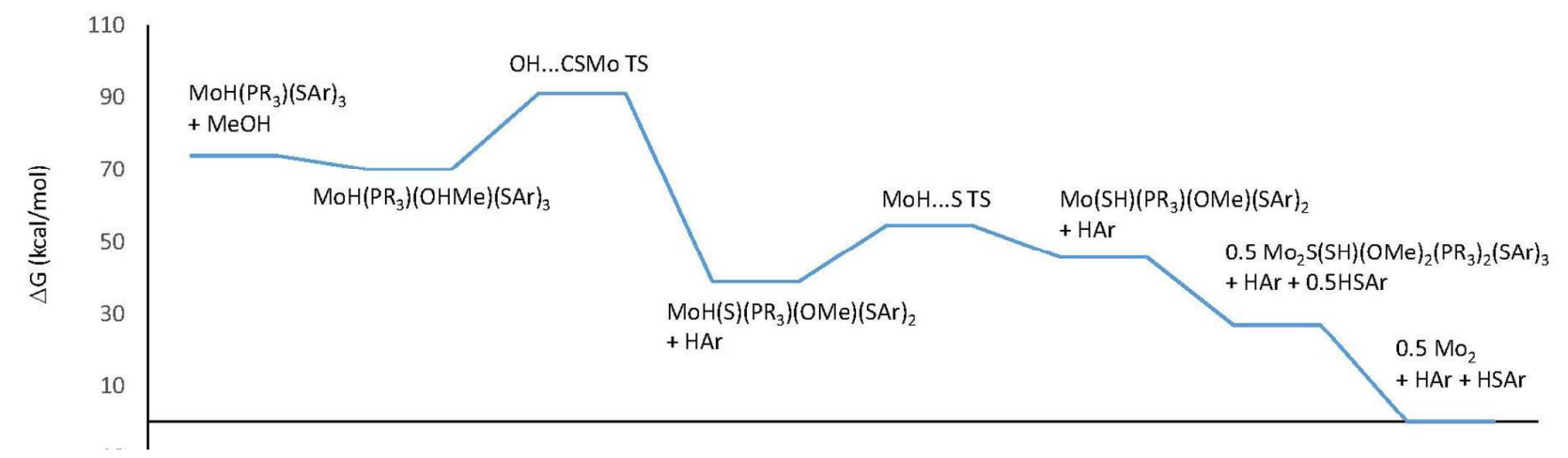

Figure 4. A reaction coordinate diagram for the C-S bond cleavage reaction shown in Figure 1. Calculations were done with M11L/6-31G(d)/SDD on Mo/THF solvation.

unprecedented mechanism: the attack by the hydrogen of the methanol ligand on the ipso carbon of an aryl (Figure 3). The result of this transfer is the formation of a $\mathrm{C}-\mathrm{H}$ bond in the arene product, consistent with the experimental observation that deuterium from $\mathrm{MeO}^{2} \mathrm{H}$ ends up on this carbon (Figure $3 b)$.

The hydrogen of the methanol ligand (Figure 3a) transfers to the aryl carbon 5 via a transition state with arenium character. Indeed the Mulliken positive charges on the carbons of the ring other than carbon 5 increase on going from the methanol adduct to the transition state. Atoms with increased negative charges include carbon 5, sulfur 4, hydrogen 16 and hydrogens on the methoxide methyl. The reaction has characteristics as described for other electrophilic aromatic substitution reactions. ${ }^{10,11}$ The immediate metal-containing product of this reaction is a formally $\mathrm{Mo}(\mathrm{VI})$ CTB sulfido complex $\mathrm{MoH}(\mathrm{S})\left(\mathrm{PR}_{3}\right)(\mathrm{OMe})(\mathrm{SAr})_{2}$ with a short Mo-S bond of $2.14 \AA$ (Figure $3 \mathrm{c}$ ). The metal undergoes a formal two electron oxidation in this reaction, paradoxically indicating that the hydrogen transferred as a hydride.

The $(\mathrm{OH} . . . \mathrm{CSMo})$ transition state $\left(1635 \mathrm{~cm}^{-1}\right)$ is the highest energy point in the overall reaction shown in Figure 1 with a transition state energy of $21 \mathrm{kcal} / \mathrm{mol}$ above the energy of the methanol adduct $\mathrm{MoH}\left(\mathrm{PR}_{3}\right)(\mathrm{OHMe})(\mathrm{SAr})_{3}$ (Figure 4); the same TS energy was obtained for gas phase and liquid phase (THF) calculations. The use of higher basis sets on the central atoms $(6-311++G(d, p))$ resulted in an energy of $22 \mathrm{kcal} / \mathrm{mol}$ and the use of the PBEO functional with dispersion correction D3 gave $24 \mathrm{kcal} / \mathrm{mol}$. Little is known about the mechanism of protonolysis of carbon-sulfur bonds. ${ }^{12,13}$

Another interesting step in the overall reaction involves the transition state for the formal reductive elimination of the $\mathrm{Mo}(\mathrm{VI}) \mathrm{CTB}$ hydridosulfido complex $\mathrm{MoH}(\mathrm{S})\left(\mathrm{PR}_{3}\right)(\mathrm{OMe})(\mathrm{SAr})_{2}$ to produce the $\mathrm{Mo}(\mathrm{IV})$ hydrosulfido complex $\mathrm{Mo}(\mathrm{SH})\left(\mathrm{PR}_{3}\right)(\mathrm{OMe})(\mathrm{SAr})_{2}$ (Figure 5$)$. This step has a barrier of $15 \mathrm{kcal} / \mathrm{mol}$ and is uphill thermodynamically by $7 \mathrm{kcal} / \mathrm{mol}$ but is likely driven by subsequent dimerization steps where HSAr is eliminated, a strongly downhill reaction (Figure 4). A similar transition state involving hydride migration to a bridging sulfido ligand in a formal hydride to proton conversion has been reported. ${ }^{6 b}$ Molybdenum hydrido sulfido complexes can also readily eliminate dihydrogen. ${ }^{14}$
The details of the subsequent steps have not been established. The reaction may proceed via a singly bridged sulfido complex such as $\mathrm{Mo}_{2}(\mu-\mathrm{S})(\mathrm{SH})(\mathrm{OMe})_{2}\left(\mathrm{PMe}_{3}\right)_{2}(\mathrm{SAr})_{3}$ (Figure 6) which might eliminate a further equivalent of thiol $\mathrm{HSAr}$ to give the observed dimeric product $\left(\mathrm{PMe}_{3}\right)(\mathrm{MeO})(\mathrm{ArS}) \mathrm{Mo}(\mu-\mathrm{S}){ }_{2} \mathrm{Mo}(\mathrm{SAr})(\mathrm{OMe})\left(\mathrm{PMe}_{3}\right) \quad\left(\mathrm{Mo}_{2}\right) \quad$ of Figure 1 found at the zero energy point of the reaction coordinate diagram, Figure 4.

These unusual reactions and geometries highlight the rich redox chemistry of molybdenum in a sulfur environment. The chemistry has possible relevance to HDS catalysis but also the action of molybdenum enzymes. ${ }^{15}$

This project was funded by an NSERC discovery grant, a Killam Research Fellowship to R. H. M. from the Canada Council for the Arts, and supported by computational resources from Sharcnet (www.sharcnet.ca), Scinet (www.scinethcp.ca) and Compute Canada (www.computecanada.ca).

\section{Conflicts of interest}

There are no conflicts to declare.
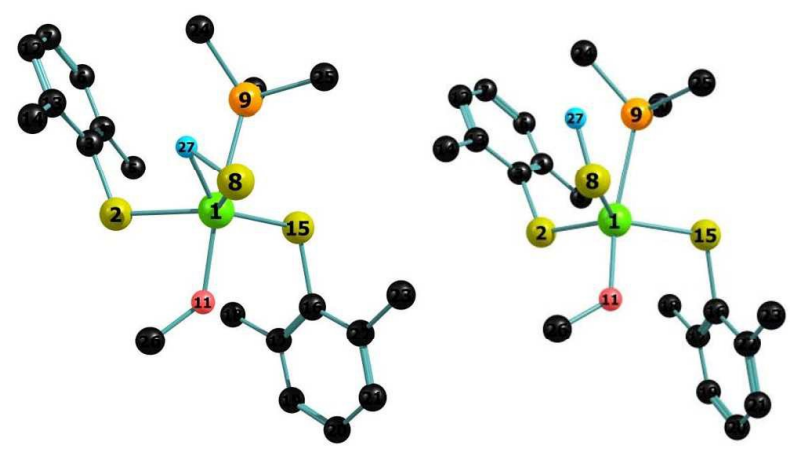

Figure 5. (a) Transition state structure MoH..S TS $\left(959 i \mathrm{~cm}^{-1}\right)$ for the hydride reductive elimination step (Mo(1)-H(27) 1.77, Mo(1)-P(9) 2.59, Mo(1)-O(11) 1.92, Mo(1)-S(2) 2.35, Mo(1)-S(8) 2.23, Mo(1)-S(15) 2.35, H(27)-S(8) $1.59 \AA$; H(27)-Mo(1)-P(9) 66.0, $\mathrm{Mo}(1)-\mathrm{H}(27)-\mathrm{S}(8)$ 82.7, P(9)-Mo(1)-O(11) 161.3, S(2)-Mo(1)-S(15) 119.8 , S(2)-Mo(1)$\left.\mathrm{S}(8) 122.3^{\circ}\right)$ to give the (b) hydrosulfido complex $\mathrm{Mo}(\mathrm{SH})\left(\mathrm{PR}_{3}\right)(\mathrm{OMe})(\mathrm{SAr})_{2}(\mathrm{Mo}(1)-\mathrm{P}(9)$ 2.52, Mo(1)-O(11) 1.92, Mo(1)-S(2) 2.30, Mo(1)-S(8) 2.39, Mo(1)-S(15) 2.29, H(27)-S(8) $1.34 \AA ̊$; P(9)-Mo(1)-O(11) 159.3, S(2)-Mo(1)-S(15) 117.1, S(2)-Mo(1)-S(8) 120.1, S(8)$\left.\mathrm{Mo}(1)-S(15) 122.8^{\circ}\right)$. 


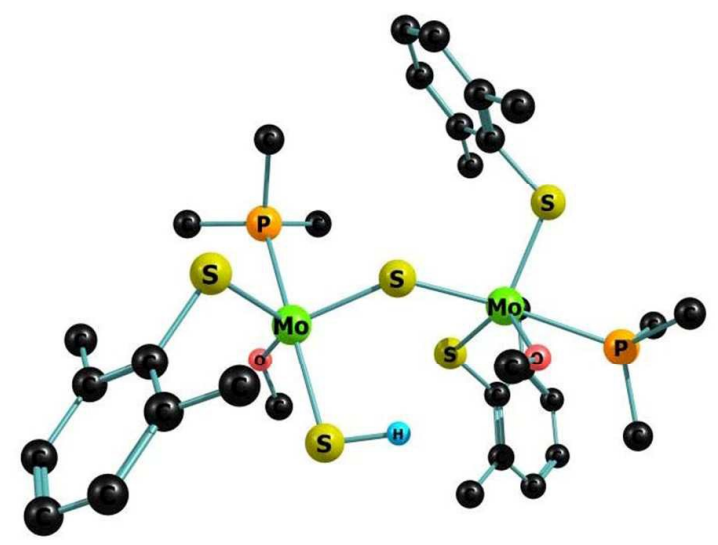

Figure 6. Structure of a possible intermediate in the reaction shown in Figure 1, a sulfido bridged dimer $\mathrm{Mo}_{2}(\mu-\mathrm{S})(\mathrm{SH})(\mathrm{OMe})_{2}\left(\mathrm{PMe}_{3}\right)_{2}(\mathrm{SAr})_{3}$.

\section{Notes and references}

1 V. C. Srivastava, RSC Adv., 2012, 2, 759-783.

2 P. Y. Prodhomme, P. Raybaud and H. Toulhoat, J. Catal., 2011, 280, 178-195.

3 S. J. Ding, S. J. Jiang, Y. S. Zhou, Q. Wei and W. W. Zhou, J. Catal., 2017, 345, 24-38.

4 L. Wang, W. He and Z. Yu, Chem. Soc. Rev., 2013, 42, 599621.

5 (a) A. Sattler, K. E. Janak and G. Parkin, Inorg. Chim. Acta, 2011, 369, 197-202; (b) K. E. Janak, J. M. Tanski, D. G. Churchill and G. Parkin, J. Am. Chem. Soc., 2002, 124, 41824183.

6 (a) A. G. Algarra, Eur. J. Inorg. Chem., 2016, 1886-1894; (b) J. E. McGrady and J. Gracia, J. Organomet. Chem., 2005, 690, 5206-5214.

7 T. E. Burrow, A. Hills, D. L. Hughes, J. D. Lane, N. J. Lazarowych, M. J. Maguire, R. H. Morris and R. L. Richards, J. Chem. Soc., Chem. Commun, 1990, 1757-1759.

8 T. E. Burrow, D. L. Hughes, A. J. Lough, M. J. Maguire, R. H. Morris and R. L. Richards, J. Chem. Soc., Dalton Trans., 1995, 2583-2589

9 R. H. Morris, Coord. Chem. Rev., 2017, DOI: https://doi.org/10.1016/i.ccr.2017.06.019.

10 B. Galabov, D. Nalbantova, P. V. Schleyer and H. F. Schaefer, Acc. Chem. Res., 2016, 49, 1191-1199.

11 S. L. C. Moors, X. Deraet, G. Van Assche, P. Geerlings and F. De Proft, Chem. Sci., 2017, 8, 680-688.

12 M. Rakowski DuBois, Polyhedron, 1997, 16, 3089-3098.

13 T. Y. Luh and Z. J. Ni, Synthesis, 1990, 89-103.

14 V. J. Murphy and G. Parkin, J. Am. Chem. Soc., 1995, 117, 3522-3528.

15 R. Hille, J. Hall and P. Basu, Chem. Rev., 2014, 114, 3963 4038. 
Page $5^{\circ}$ of 5

$\rightarrow H$

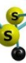

$+\mathrm{MeOH} \rightarrow$

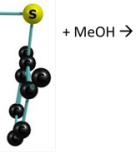

chenfícomm

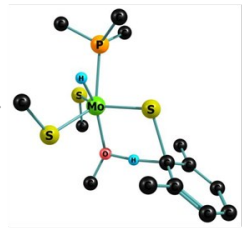

A five coordinate molybdenum hydride with an unprecedented CTP structure reacts with methanol, leading to the facile cleavage of a sulfur-carbon bond. 


\section{Supplementary Information for:}

\section{A capped trigonal pyramidal molybdenum hydrido complex and an unusually mild sulfur-carbon bond cleavage reaction}

Robert H. Morris

Contents

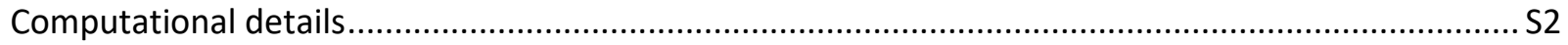

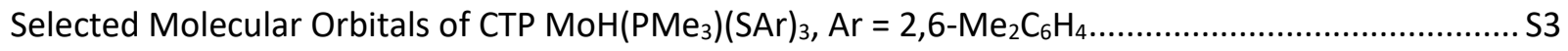

Calculation of ${ }^{2} \mathrm{~J}_{\mathrm{PH}}$ coupling constants of $\mathrm{CTP} \mathrm{MoH}\left(\mathrm{PMe}_{3}\right)(\mathrm{SAr})_{3}$ and the more stable trans isomer 2, trans- $\mathrm{MoH}\left(\mathrm{PMe}_{3}\right)(\mathrm{SAr})_{3}, \mathrm{Ar}=2,6-\mathrm{Me}_{2} \mathrm{C}_{6} \mathrm{H}_{3}$ using the GIAO method ..............................................S6

Energies (in Hartrees), Structures and Cartesian coordinates.......................................................... 88

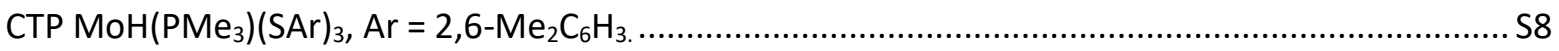

$\mathrm{MeOH}$

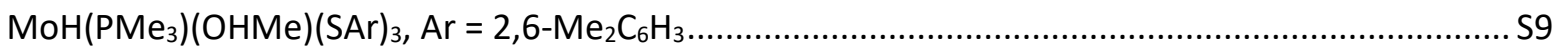

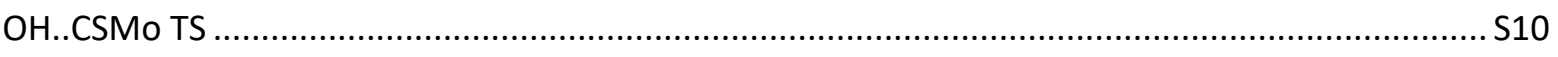

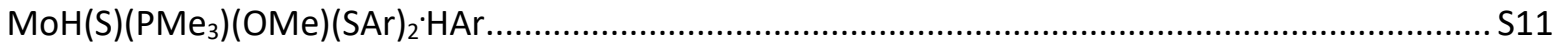

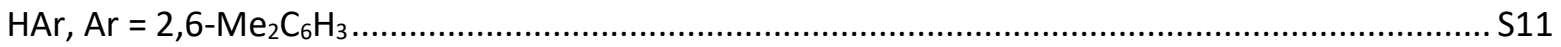

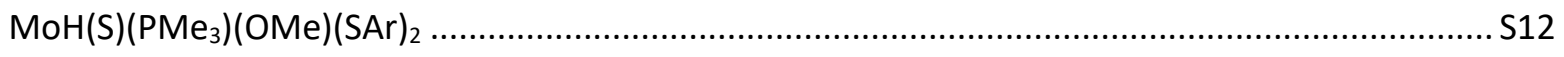

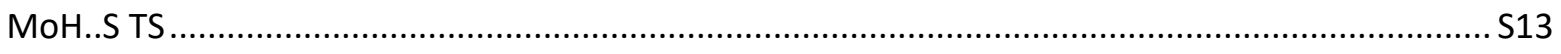

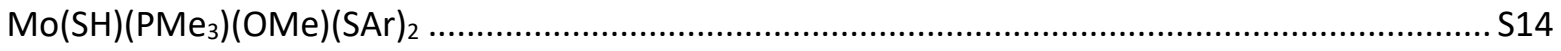

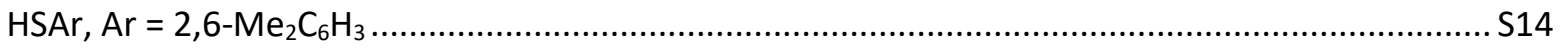

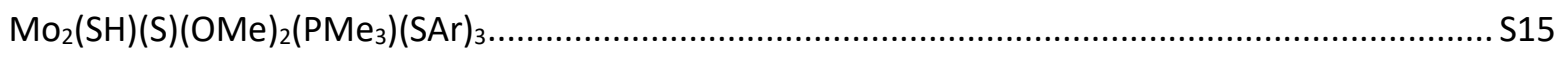

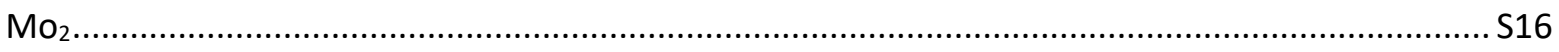

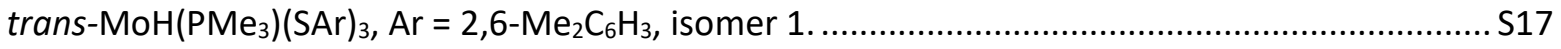

trans-MoH( $\left(\mathrm{PMe}_{3}\right)(\mathrm{SAr})_{3}, \mathrm{Ar}=2,6-\mathrm{Me}_{2} \mathrm{C}_{6} \mathrm{H}_{3}$, isomer 2........................................................ 18

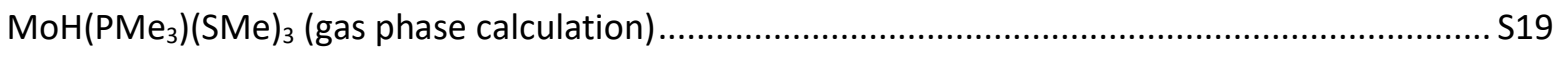

trans-MoH($\left(\mathrm{PMe}_{3}\right)(\mathrm{SMe})_{3}$ (gas phase calculation) …................................................................... S19 


\section{Computational details}

Density functional theory calculations were performed using the Gaussian09 package $^{1}$ and the M11-L functional unless otherwise noted. All of the atoms were treated with the 6-31+G(d,p) basis set (BS1) except for the molybdenum which was treated with the Stuttgart effective core potential (SDD). An ultrafine grid (pruned: 99,590) and the integral equation formalism polarizable continuum model (IEFPCM) with radii and nonelectrostatic terms from the SMD solvation model for THF (scrf=(smd,solvent=tetrahydrofuran) $)$ were used for all optimizations. Ground states were connected to their transition states by performing intrinsic reaction coordinate (IRC) calculations. A frequency analysis (temperature $298 \mathrm{~K}$ ) was performed on all geometries and this produced no imaginary frequencies for the ground state structures and only one imaginary frequency for transition states. The geometries of the three structures involved in the $\mathrm{OH}$... CSMo transition state (Figure 3 of the article) were also calculated using a higher basis set 6-311++G(d,p) (BS2) on all of the atoms attached to molybdenum as well as the ipso carbon on the cleaved arene ring. These three structures were also found with the PBE1PBE functional with the same basis set as mentioned in the article. The same transition state structure was located with one imaginary frequency. Similarly the PBE0 functional (Gaussian key word PBE1PBE) with D3 was applied to the calculation of the three structures with comparable results. Three-dimensional visualization of optimized geometries were constructed using ChemCraft.

\footnotetext{
${ }^{1}$ Gaussian 09, Revision B.01, M. J. Frisch, G. W. Trucks, H. B. Schlegel, G. E. Scuseria, M. A. Robb, J. R. Cheeseman, G. Scalmani, V. Barone, B. Mennucci, G. A. Petersson, H. Nakatsuji, M. Caricato, X. Li, H. P. Hratchian, A. F. Izmaylov, J. Bloino, G. Zheng, J. L. Sonnenberg, M. Hada, M. Ehara, K. Toyota, R. Fukuda, J. Hasegawa, M. Ishida, T. Nakajima, Y. Honda, O. Kitao, H. Nakai, T. Vreven, J. A. Montgomery, Jr., J. E. Peralta, F. Ogliaro, M. Bearpark, J. J. Heyd, E. Brothers, K. N. Kudin, V. N. Staroverov, R. Kobayashi, J. Normand, K. Raghavachari, A. Rendell, J. C. Burant, S. S. Iyengar, J. Tomasi, M. Cossi, N. Rega, J. M. Millam, M. Klene, J. E. Knox, J. B. Cross, V. Bakken, C. Adamo, J. Jaramillo, R. Gomperts, R. E. Stratmann, O. Yazyev, A. J. Austin, R. Cammi, C. Pomelli, J. W. Ochterski, R. L. Martin, K. Morokuma, V. G. Zakrzewski, G. A. Voth, P. Salvador, J. J. Dannenberg, S. Dapprich, A. D. Daniels, Ö. Farkas, J. B. Foresman, J. V. Ortiz, J. Cioslowski, and D. J. Fox, Gaussian, Inc., Wallingford CT, 2010.
} 
Selected Molecular Orbitals of CTP MoH(PMe $)_{3}(\mathrm{SAr})_{3}, \mathrm{Ar}=2,6-\mathrm{Me}_{2} \mathrm{C}_{6} \mathrm{H}_{4}$

\section{LUMO+4}

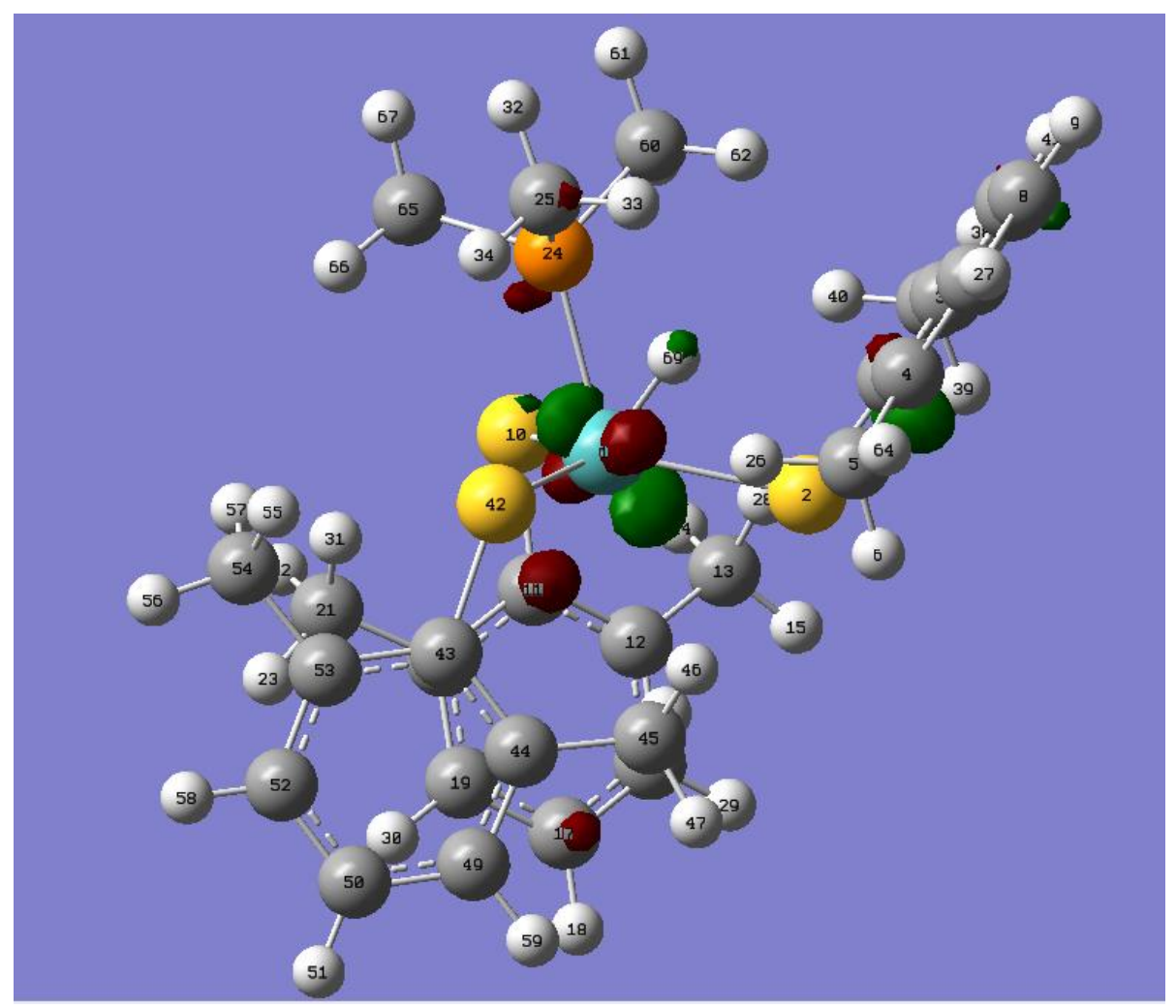

Approx. $d_{y z}$ oriented between $\operatorname{MoS}(10)$ (arene down) (approx. y direction) along Mo-P(24) (approx. $z$ direction). Some Mo-S and Mo-H antibonding. 


\section{LUMO+2}

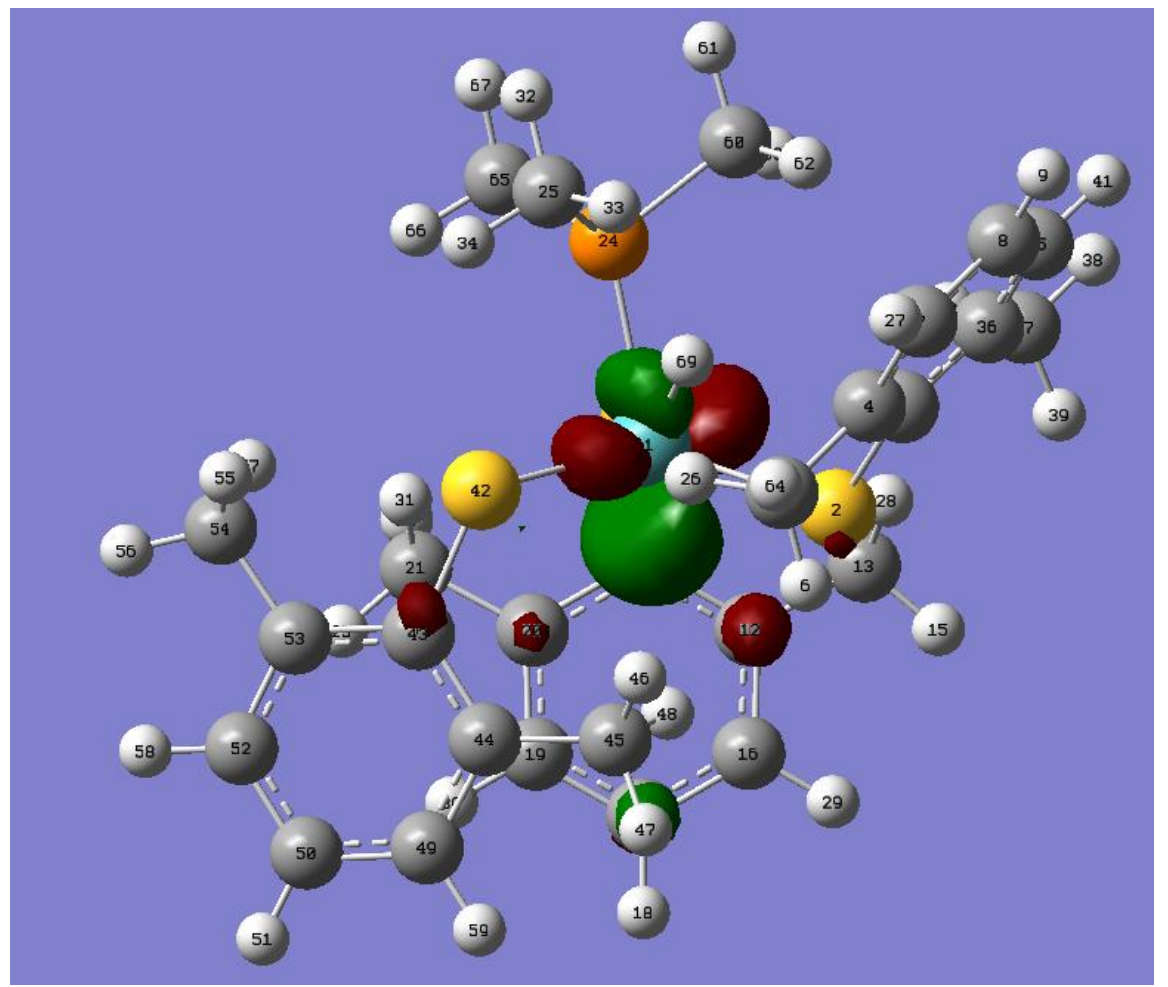

Approx. $d_{x 2-z 2}$ oriented along Mo-S(42) (x direction) and between Mo-H(69) and MoP(24) (z direction). Some Mo-S and Mo-P antibonding. 


\section{LUMO+1}

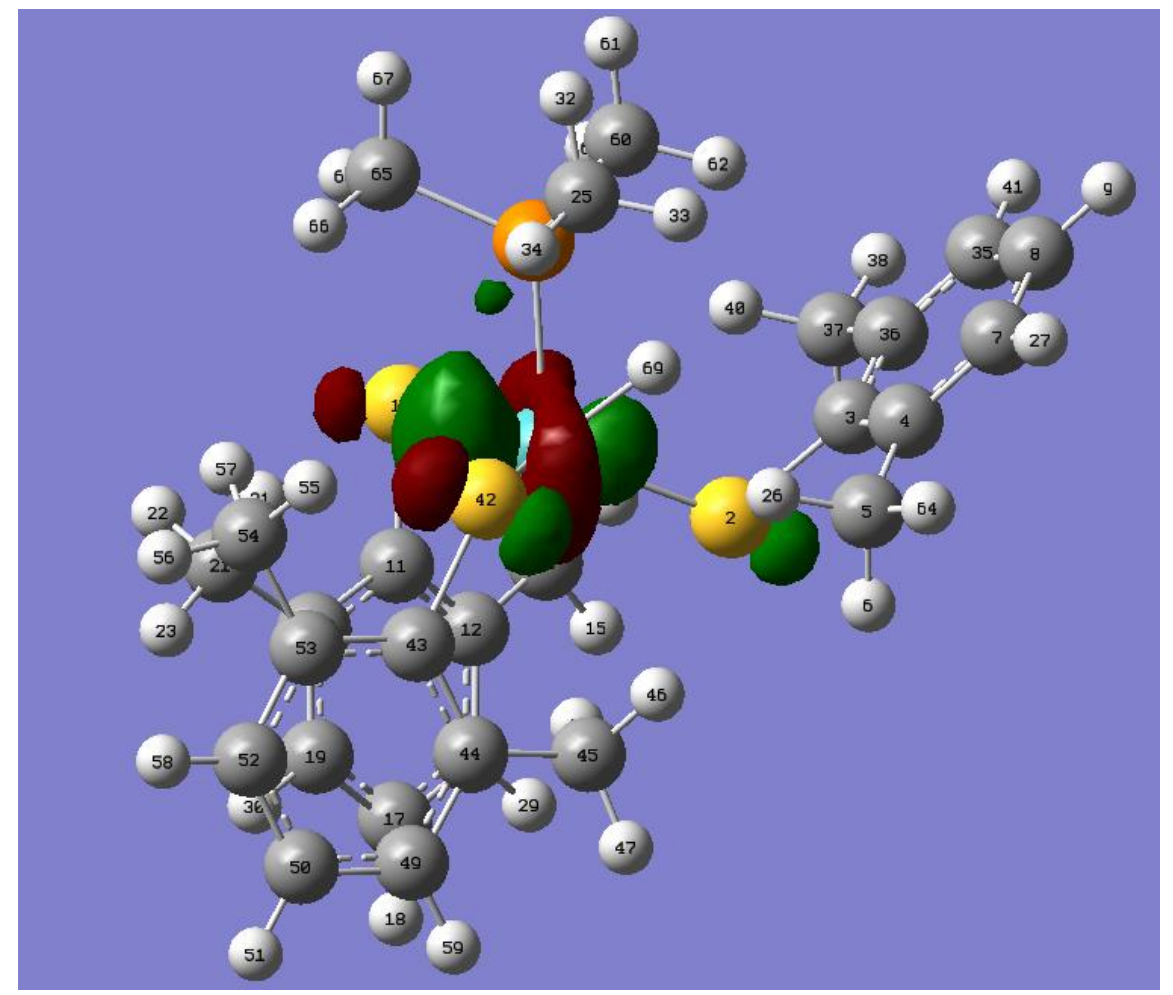

$d_{x y}+d_{x 2-y 2}$ oriented between Mo-S(10) and Mo-S(42) bond in the MoS 3 plane.

\section{LUMO}

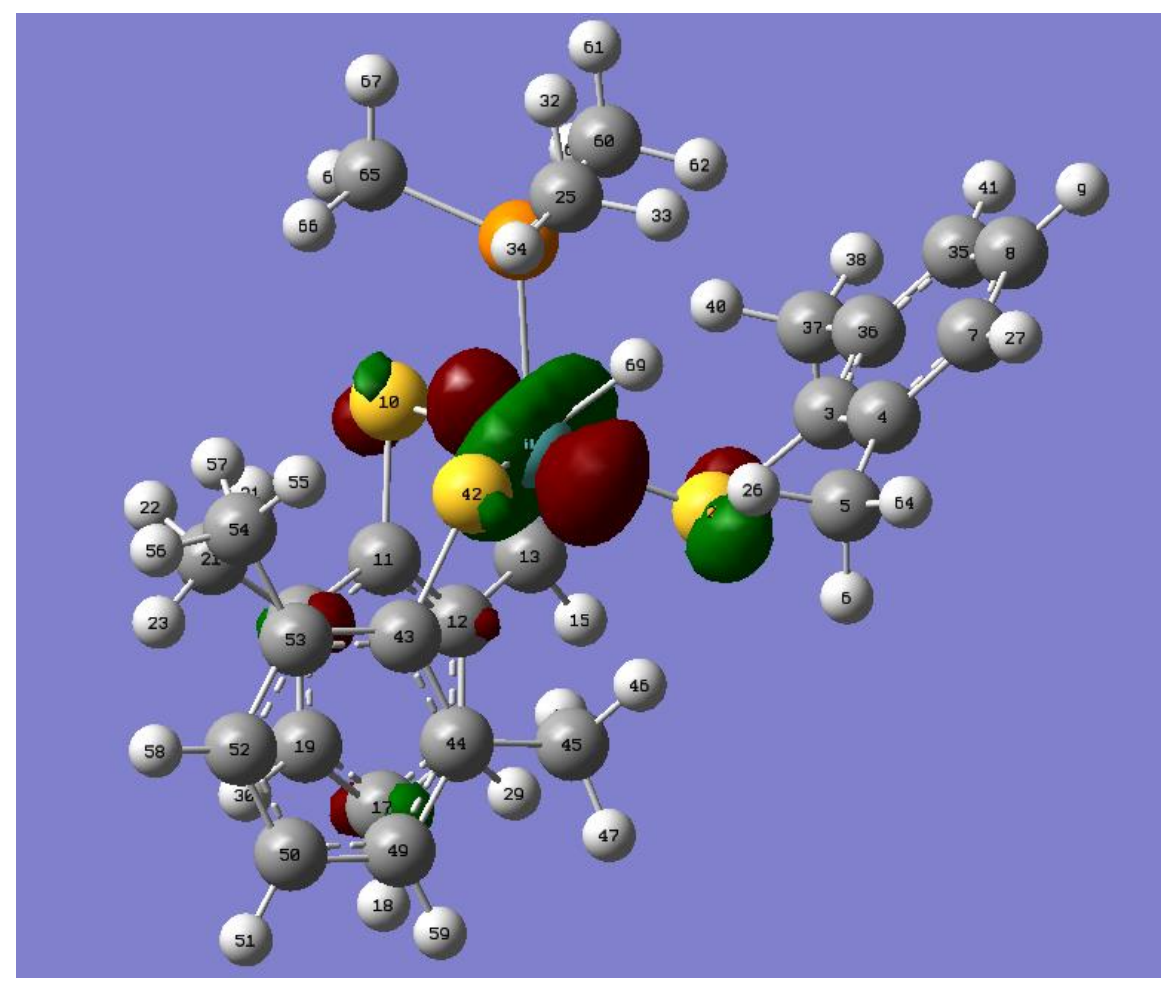


$d_{x y}-d_{x 2-y_{2}}$ oriented parallel to $S(10)$ and $S(42)$ (approx. $y$ direction) in the $\mathrm{MoS}_{3}$ plane.

HOMO

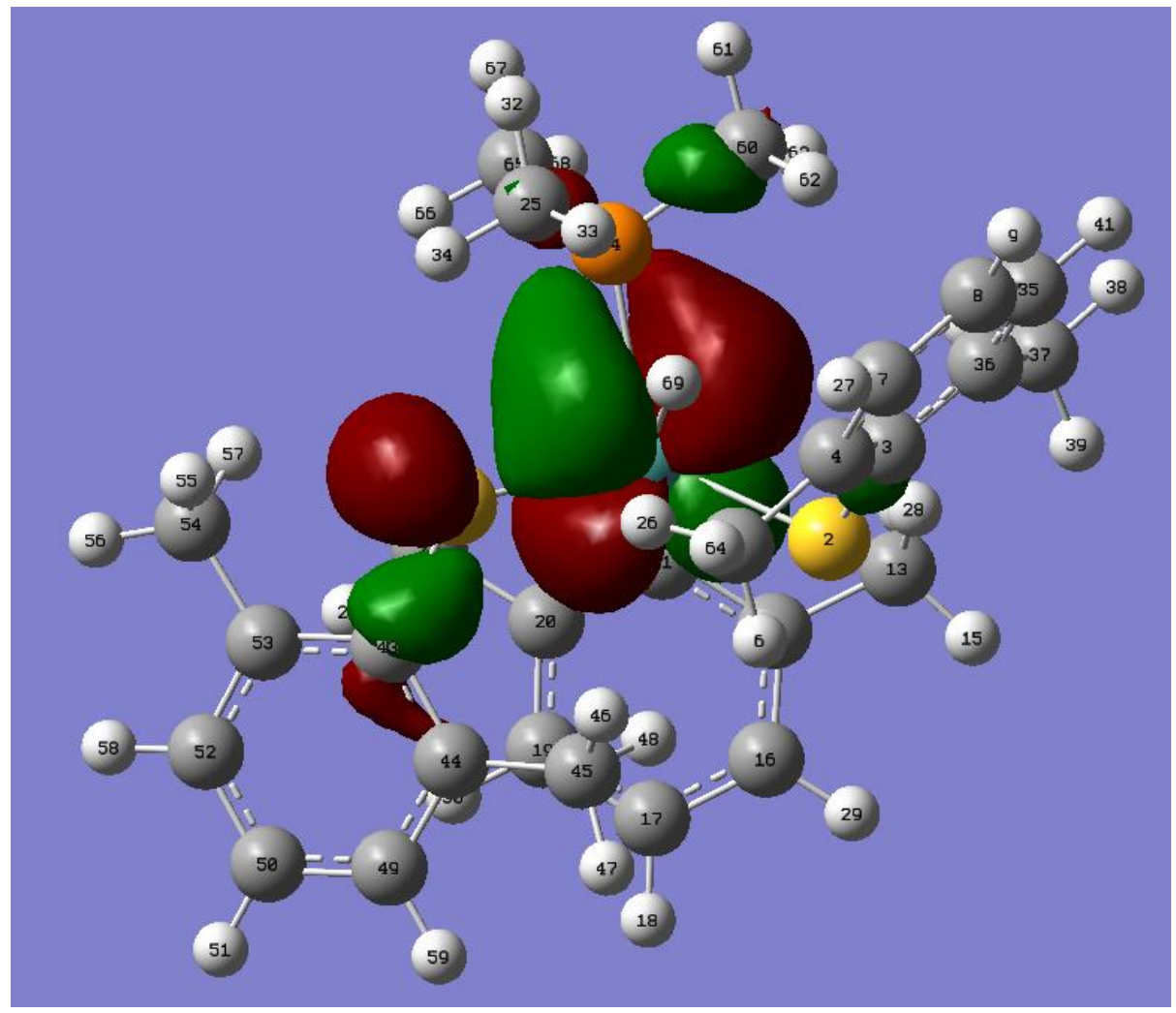

$\left(d_{x z}\right)^{2}$ between bonds Mo-S(42) (arene down) (approx. $x$ direction) and Mo-P (approx. $z$ direction). HOMO-1 to HOMO-3 were associated with the thiolate ligands (sulfur $\mathrm{p}$ and attached arene).

Calculation of ${ }^{2} \mathrm{JPH}_{\mathrm{PH}}$ coupling constants of CTP $\mathrm{MoH}\left(\mathrm{PMe}_{3}\right)(\mathrm{SAr})_{3}$ and the more stable trans isomer 2, trans-MoH( $\left.\mathrm{PMe}_{3}\right)(\mathrm{SAr})_{3}, \mathrm{Ar}=2,6-\mathrm{Me}_{2} \mathrm{C}_{6} \mathrm{H}_{3}$ using the GIAO method

\begin{tabular}{|l|l|l|}
\hline & $\begin{array}{l}{ }^{2} \mathrm{~J}_{P H}(\mathrm{~Hz}) \text { of CTP } \\
\mathrm{MoH}\left(\mathrm{PMe}_{3}\right)(\mathrm{SAr})_{3}{ }^{\mathrm{a}}\end{array}$ & $\begin{array}{l}{ }^{2} \mathrm{~J}_{\mathrm{PH}}(\mathrm{Hz}) \text { of isomer 2, } \\
\text { trans-MoH(PMe })_{3}(\mathrm{SAr})_{3}{ }^{a}\end{array}$ \\
\hline $\begin{array}{l}\text { Fermi Contact (FC) } \\
\text { contribution to J }\end{array}$ & -70.2 & -30.7 \\
\hline $\begin{array}{l}\text { Spin-dipolar (SD) } \\
\text { contribution to J }\end{array}$ & -0.2 & -0.5 \\
\hline $\begin{array}{l}\text { Paramagnetic spin-orbit } \\
\text { (PSO) contribution to J }\end{array}$ & -0.8 & -0.7 \\
\hline
\end{tabular}




\begin{tabular}{|l|l|l|}
\hline $\begin{array}{l}\text { Diamagnetic spin-orbit } \\
\text { (DSO) contribution to J }\end{array}$ & 1.7 & 0.2 \\
\hline $\begin{array}{l}\text { Total nuclear spin-spin } \\
\text { coupling J }\end{array}$ & -69.5 & -31.9 \\
\hline
\end{tabular}

${ }^{a}$ Gaussian input line: \# NMR=(MIXED,READATOMS,FCOnly) m11l/genecp int=(ultrafine, acc2e=11) scrf=(smd, solvent=tetrahydrofuran) with 6-31G* on H C S P and SDD on Mo. 
Energies (in Hartrees), Structures and Cartesian coordinates

CTP MoH( $\left.\mathrm{PMe}_{3}\right)(\mathrm{SAr})_{3}, \mathrm{Ar}=2,6-\mathrm{Me}_{2} \mathrm{C}_{6} \mathrm{H}_{3}$

\section{Enthalpy -2654.469362}

\section{Free energy -2654.602966}

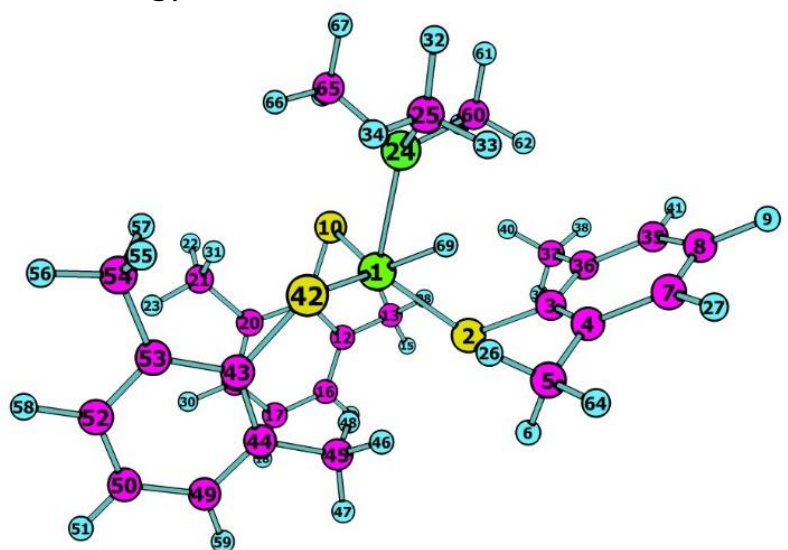

$\begin{array}{lrr}\text { Mo } & 0.250922 & -0.053210 \\ \mathrm{~S} & 1.585512 & 1.000625 \\ \mathrm{C} & 3.185318 & 0.267682 \\ \mathrm{C} & 3.357194 & -0.901864 \\ \mathrm{C} & 2.220618 & -1.557259 \\ \mathrm{H} & 1.684778 & -0.853756 \\ \mathrm{C} & 4.632437 & -1.449867 \\ \mathrm{C} & 5.712270 & -0.854437 \\ \mathrm{H} & 6.709171 & -1.299384 \\ \mathrm{~S} & -0.309576 & 1.365466 \\ \mathrm{C} & -1.410925 & 2.139329 \\ \mathrm{C} & -0.978719 & 3.257695 \\ \mathrm{C} & 0.333654 & 3.906508 \\ \mathrm{H} & 0.402556 & 4.274515 \\ \mathrm{H} & 0.477550 & 4.765412 \\ \mathrm{C} & -1.839899 & 3.787922 \\ \mathrm{C} & -3.103617 & 3.257958 \\ \mathrm{H} & -3.763854 & 3.687799 \\ \mathrm{C} & -3.544679 & 2.211035 \\ \mathrm{C} & -2.723651 & 1.642548 \\ \mathrm{C} & -3.265685 & 0.584575 \\ \mathrm{H} & -3.291946 & 0.931266 \\ \mathrm{H} & -4.291554 & 0.316081 \\ \mathrm{P} & 1.439837 & -1.290399 \\ \mathrm{C} & 1.658118 & -3.053146 \\ \mathrm{H} & 1.464458 & -1.948332 \\ \mathrm{H} & 4.774267 & -2.365996 \\ \mathrm{H} & 1.181979 & 3.217955 \\ \mathrm{H} & -1.508241 & 4.651317 \\ \mathrm{H} & -4.557514 & 1.811822 \\ \mathrm{H} & -2.658263 & -0.334183 \\ \mathrm{H} & 2.230429 & -3.532172 \\ \mathrm{H} & 2.194808 & -3.210719\end{array}$

$\begin{array}{rrrr}\text { H } & 0.683216 & -3.559636 & 1.623056 \\ \mathrm{C} & 5.530548 & 0.311427 & -0.877083 \\ \mathrm{C} & 4.274321 & 0.894786 & -0.758428 \\ \mathrm{C} & 4.107182 & 2.137794 & 0.041295 \\ \mathrm{H} & 5.069570 & 2.464650 & 0.463648 \\ \mathrm{H} & 3.704341 & 2.969617 & -0.561390 \\ \mathrm{H} & 3.398323 & 1.998737 & 0.877186 \\ \mathrm{H} & 6.384317 & 0.791924 & -0.384580 \\ \mathrm{~S} & -1.057087 & -1.902434 & -0.137156 \\ \mathrm{C} & -2.646507 & -1.528020 & -0.843406 \\ \mathrm{C} & -2.818545 & -0.660316 & -1.932986 \\ \mathrm{C} & -1.688177 & 0.076132 & -2.555419 \\ \mathrm{H} & -0.804548 & -0.561308 & -2.721378 \\ \mathrm{H} & -1.986775 & 0.505480 & -3.524304 \\ \mathrm{H} & -1.354524 & 0.927752 & -1.930893 \\ \mathrm{C} & -4.099471 & -0.462801 & -2.437044 \\ \mathrm{C} & -5.196391 & -1.096895 & -1.886866 \\ \mathrm{H} & -6.196927 & -0.927044 & -2.297893 \\ \mathrm{C} & -5.016951 & -1.957933 & -0.820368 \\ \mathrm{C} & -3.754925 & -2.196157 & -0.290633 \\ \mathrm{C} & -3.616591 & -3.135119 & 0.855684 \\ \mathrm{H} & -2.955108 & -3.986051 & 0.621775 \\ \mathrm{H} & -4.597427 & -3.542749 & 1.144686 \\ \mathrm{H} & -3.179185 & -2.647192 & 1.744190 \\ \mathrm{H} & -5.878553 & -2.473027 & -0.378814 \\ \mathrm{H} & -4.227800 & 0.216022 & -3.288989 \\ \mathrm{C} & 3.128055 & -0.742233 & 2.342670 \\ \mathrm{H} & 3.598770 & -1.354405 & 3.130311 \\ \mathrm{H} & 3.739358 & -0.822513 & 1.429104 \\ \mathrm{H} & 3.137295 & 0.309480 & 2.670098 \\ \mathrm{H} & 2.572414 & -2.399711 & -3.452311 \\ \mathrm{C} & 0.726437 & -1.335050 & 3.686223 \\ \mathrm{H} & -0.321695 & -1.672246 & 3.647787 \\ \mathrm{H} & 1.293160 & -2.032201 & 4.325923 \\ \mathrm{H} & 0.744042 & -0.343332 & 4.163018 \\ \mathrm{H} & 1.522741 & -1.067323 & 0.097938 \\ \mathrm{M} & & & \end{array}$

$\mathrm{MeOH}$

\section{Enthalpy -115.618887 Free energy -115.64534}

$\begin{array}{rrrr}\mathrm{C} & -0.678322 & 0.018693 & 0.000000 \\ \mathrm{H} & -1.023161 & 0.224198 & 1.015409 \\ \mathrm{H} & -1.138490 & -0.903313 & -0.361043 \\ \mathrm{H} & -0.959528 & 0.846298 & -0.654365 \\ \mathrm{O} & 0.754203 & -0.127836 & 0.000000 \\ \mathrm{H} & 1.157490 & 0.743347 & 0.000000\end{array}$


$\mathrm{MoH}\left(\mathrm{PMe}_{3}\right)(\mathrm{OHMe})(\mathrm{SAr})_{3}, \mathrm{Ar}=2,6-\mathrm{Me}_{2} \mathrm{C}_{6} \mathrm{H}_{3}$

Enthalpy -2770.137055
Free energy -2770.254194

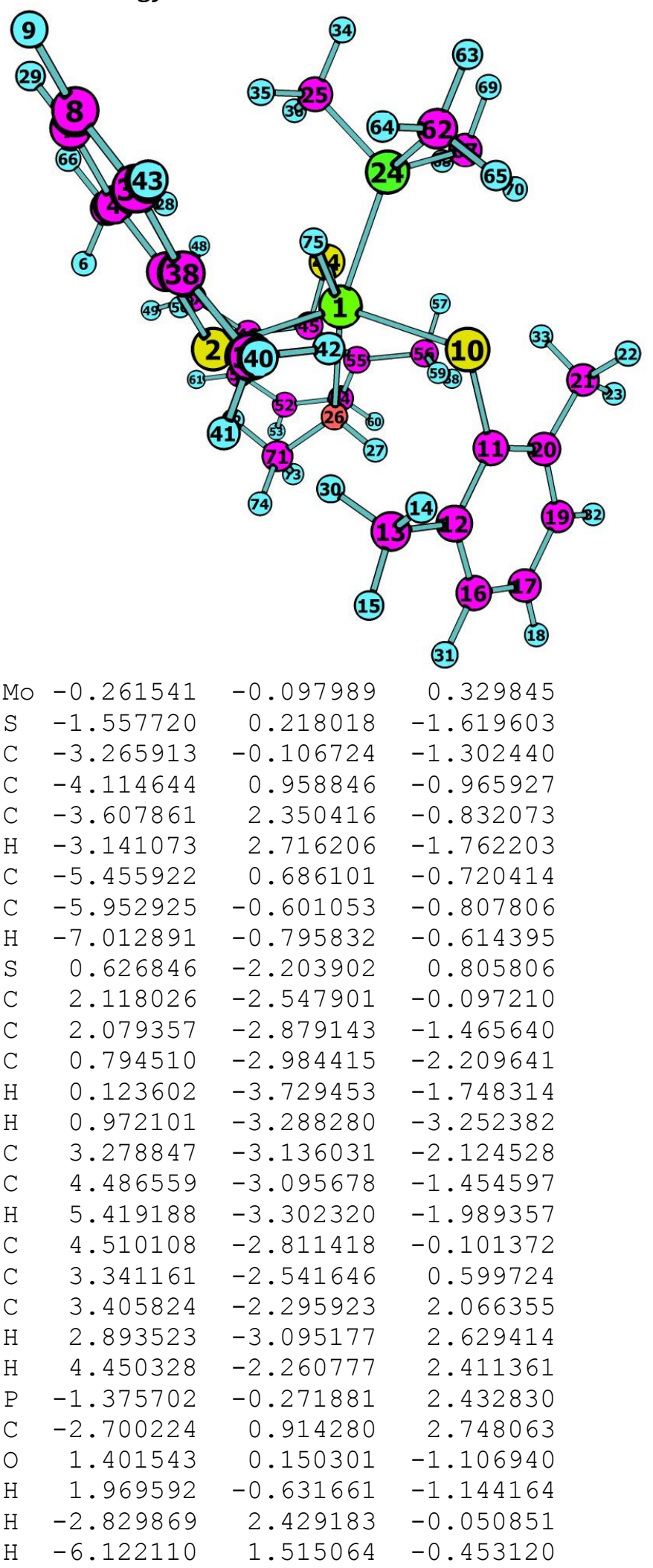

$\begin{array}{rrr}0.223951 & -2.039560 & -2.225519 \\ 3.250429 & -3.387462 & -3.191388 \\ 5.463702 & -2.802738 & 0.439685 \\ 2.914494 & -1.356155 & 2.366628 \\ -3.132995 & 0.779825 & 3.753582 \\ -3.505123 & 0.786877 & 2.004682 \\ -2.325688 & 1.947399 & 2.668454 \\ -5.105583 & -1.640998 & -1.142442 \\ -3.757097 & -1.418076 & -1.397134 \\ -2.865948 & -2.561843 & -1.723603 \\ -3.440190 & -3.497764 & -1.799089 \\ -2.326963 & -2.412834 & -2.674667 \\ -2.086505 & -2.702841 & -0.952083 \\ -5.492937 & -2.664413 & -1.213212 \\ 0.437267 & 1.947349 & 1.270133 \\ 1.655146 & 2.749346 & 0.264978 \\ 1.246450 & 3.771123 & -0.606486 \\ -0.190171 & 4.104856 & -0.796197 \\ -0.679671 & 4.408378 & 0.144494 \\ -0.310533 & 4.925243 & -1.520228 \\ -0.759641 & 3.234454 & -1.168402 \\ 2.213228 & 4.453167 & -1.336676 \\ 3.554388 & 4.143185 & -1.213470 \\ 4.302878 & 4.692420 & -1.794202 \\ 3.945777 & 3.136807 & -0.349149 \\ 3.014513 & 2.429247 & 0.401378 \\ 3.469673 & 1.332872 & 1.293961 \\ 3.100870 & 1.450322 & 2.326836 \\ 4.568635 & 1.276117 & 1.325313 \\ 3.093026 & 0.357488 & 0.941841 \\ 5.008132 & 2.885239 & -0.244607 \\ 1.893556 & 5.247789 & -2.021558 \\ -2.182974 & -1.844416 & 2.806454 \\ -2.630584 & -1.833405 & 3.813570 \\ -2.978600 & -2.051510 & 2.072424 \\ -1.462172 & -2.676444 & 2.762788 \\ -4.423927 & 3.040694 & -0.569292 \\ -0.315147 & -0.073265 & 3.891970 \\ 0.098163 & 0.944708 & 3.947271 \\ -0.880111 & -0.272867 & 4.818172 \\ 0.531332 & -0.777885 & 3.845345 \\ 1.524610 & 0.845205 & -2.322209 \\ 0.814574 & 1.681278 & -2.304382 \\ 2.547699 & 1.241250 & -2.434152 \\ 1.284918 & 0.190532 & -3.178671 \\ -1.820127 & -0.584685 & 0.619321\end{array}$


OH..CSMO TS

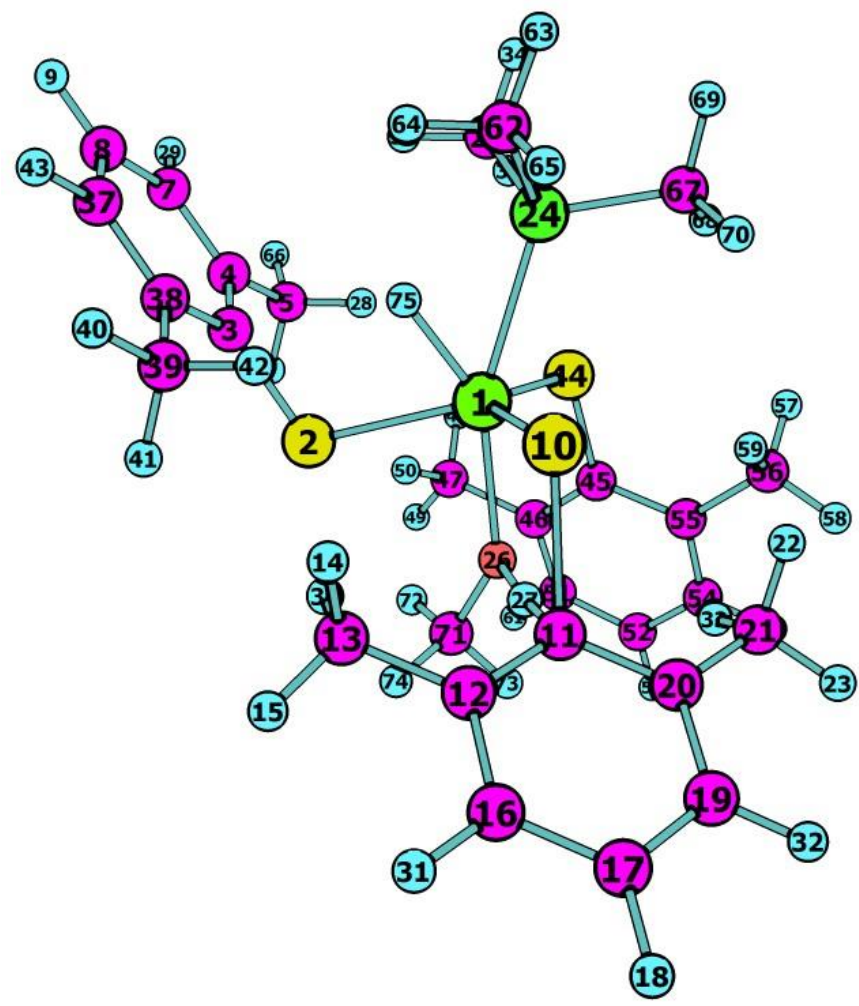

Enthalpy -2770.10478

Free energy -2770.220971

$\begin{array}{lrr}\text { Mo } & -0.283338 & 0.000257 \\ \text { S } & -1.517255 & -0.283532 \\ \mathrm{C} & -3.240396 & -0.414344 \\ \mathrm{C} & -4.024603 & 0.749118 \\ \mathrm{C} & -3.438357 & 2.097859 \\ \mathrm{H} & -2.961443 & 2.189530 \\ \mathrm{C} & -5.379720 & 0.624710 \\ \mathrm{C} & -5.951031 & -0.611216 \\ \mathrm{H} & -7.020677 & -0.688947 \\ \mathrm{~S} & 0.710540 & -1.756988 \\ \mathrm{C} & 2.297277 & -2.302903 \\ \mathrm{C} & 1.974075 & -3.462912 \\ \mathrm{C} & 0.606719 & -3.666602 \\ \mathrm{H} & -0.063824 & -4.103310 \\ \mathrm{H} & 0.615017 & -4.357652 \\ \mathrm{C} & 2.951900 & -4.422999 \\ \mathrm{C} & 4.223239 & -4.273394 \\ \mathrm{H} & 4.979986 & -5.046731 \\ \mathrm{C} & 4.543555 & -3.141362 \\ \mathrm{C} & 3.597585 & -2.155475 \\ \mathrm{C} & 3.963862 & -0.921540 \\ \mathrm{H} & 3.411804 & -0.830392 \\ \mathrm{H} & 5.039484 & -0.889262 \\ \mathrm{P} & -1.444328 & 0.376880\end{array}$

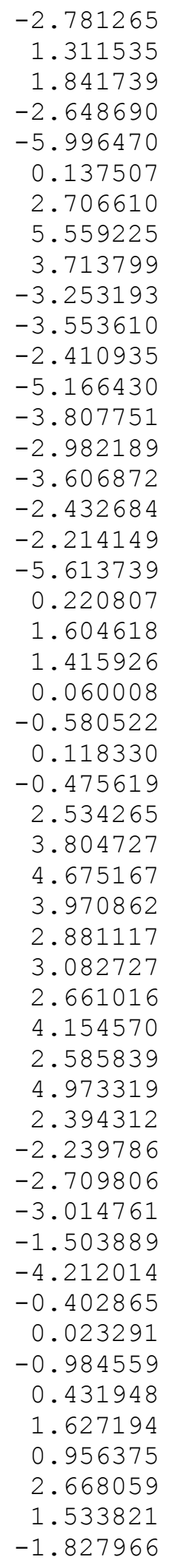

1.585948

$-0.121255$

$-1.159571$

2. 328845

1.530632

$-2.721167$

$-5.310376$

$-3.011766$

$-0.024226$

1.700341

1.262214

2.572378

$-1.748934$

$-1.675641$

$-2.911207$

$-3.803068$

$-3.042164$

$-2.887707$

$-2.731867$

2.293913

2.797745

3.290009

3.408421

4.101562

3.782709

2.443866

3.676481

3.588457

3.886842

3.145390

2.765243

2.382526

3.145251

2.287094

1.435891

3. 105197

4.052803

$-1.072402$

$-0.829929$

$-1.461462$

$-1.875418$

2. 877132

0.942608

1.934353

1.000913

0.240466

0.090896

0.838724

0.456410

$-0.842734$

$-0.490502$
2.439582

$-0.953396$

$-0.614878$

$-0.714904$

$-0.910576$

$-1.702537$

$-1.654978$

0.593826

0.575448

3.429318

1.721832

2.119155

$-0.748670$

$-1.032958$

$-1.037647$

$-0.877439$

$-1.985207$

$-0.242721$

$-0.558435$

0.772431

$-0.196309$

$-1.495341$

$-2.093761$

$-1.521098$

$-3.127356$

$-2.107033$

$-2.225483$

$-1.687799$

$-2.281326$

$-0.387550$

0.386676

1.810768

2.489315

2.044742

2.076098

0.055331

$-3.245949$

3.205503

4.172382

2. 526110

3. 370357

$-1.377642$

3. 854706

3.643472

4.789919

4.010556

$-2.282055$

$-2.734580$

$-2.375282$

$-2.873282$

0.726101 


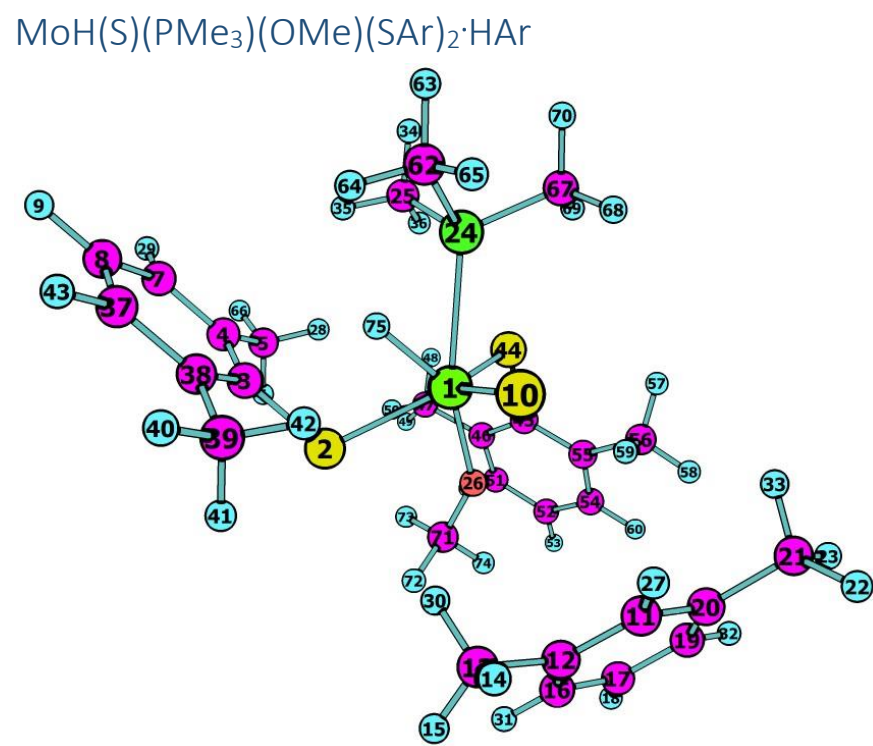

$\begin{array}{rrrr}\mathrm{H} & -3.764864 & 1.702538 & 1.233994 \\ \mathrm{H} & -2.511770 & 2.897215 & 1.674537 \\ \mathrm{C} & -5.218435 & -2.020928 & -0.978067 \\ \mathrm{C} & -3.843243 & -1.934384 & -1.164060 \\ \mathrm{C} & -2.995107 & -3.145981 & -1.019385 \\ \mathrm{H} & -3.611352 & -4.035710 & -0.820743 \\ \mathrm{H} & -2.392389 & -3.342710 & -1.921453 \\ \mathrm{H} & -2.271161 & -3.042285 & -0.190044 \\ \mathrm{H} & -5.659546 & -2.997417 & -0.745559 \\ \mathrm{~S} & 0.321607 & 2.119447 & 1.021201 \\ \mathrm{C} & 1.683049 & 2.504092 & -0.027726 \\ \mathrm{C} & 1.473741 & 3.282747 & -1.173687 \\ \mathrm{C} & 0.115639 & 3.729934 & -1.582061 \\ \mathrm{H} & -0.398769 & 4.301241 & -0.791636 \\ \mathrm{H} & 0.164464 & 4.363009 & -2.481027 \\ \mathrm{H} & -0.539255 & 2.869543 & -1.813991 \\ \mathrm{C} & 2.571198 & 3.599616 & -1.966478 \\ \mathrm{C} & 3.837980 & 3.148573 & -1.646592 \\ \mathrm{H} & 4.689980 & 3.404843 & -2.284970 \\ \mathrm{C} & 4.026097 & 2.372153 & -0.517186 \\ \mathrm{C} & 2.962730 & 2.041262 & 0.313263 \\ \mathrm{C} & 3.189738 & 1.179106 & 1.500866 \\ \mathrm{H} & 2.789960 & 1.626575 & 2.426201 \\ \mathrm{H} & 4.264473 & 0.988569 & 1.648003 \\ \mathrm{H} & 2.686429 & 0.202882 & 1.379544 \\ \mathrm{H} & 5.027603 & 2.004523 & -0.262256 \\ \mathrm{H} & 2.415225 & 4.210418 & -2.863565 \\ \mathrm{C} & -3.043072 & -0.638660 & 2.994251 \\ \mathrm{H} & -3.693785 & -0.251910 & 3.795079 \\ \mathrm{H} & -3.676578 & -1.009577 & 2.172361 \\ \mathrm{H} & -2.468075 & -1.488764 & 3.391045 \\ \mathrm{H} & -4.316043 & 2.557604 & -1.959215 \\ \mathrm{C} & -1.079923 & 1.170453 & 3.903591 \\ \mathrm{H} & -0.340771 & 0.409165 & 4.197682 \\ \mathrm{H} & -0.549306 & 2.121450 & 3.755152 \\ \mathrm{H} & -1.800638 & 1.295416 & 4.728550 \\ \mathrm{C} & 1.343631 & 0.082786 & -2.095514 \\ \mathrm{H} & 1.183476 & -0.874660 & -2.637233 \\ \mathrm{H} & 0.771260 & 0.862333 & -2.643580 \\ \mathrm{H} & 2.417720 & 0.342080 & -2.178877 \\ \mathrm{H} & -2.136171 & -0.304127 & 0.550760 \\ \mathrm{H} \mathrm{r} & -1.56 & \end{array}$

\section{Enthalpy -2459.508987}

\section{Free energy -2459.60867}

$\begin{array}{lrr}\text { Mo } & -0.503964 & -0.072229 \\ \mathrm{~S} & -1.541830 & -0.539147 \\ \mathrm{C} & -3.286125 & -0.680098 \\ \mathrm{C} & -4.094524 & 0.461923 \\ \mathrm{C} & -3.520429 & 1.802627 \\ \mathrm{H} & -2.933526 & 1.810709 \\ \mathrm{C} & -5.464065 & 0.325249 \\ \mathrm{C} & -6.025745 & -0.903564 \\ \mathrm{H} & -7.107177 & -0.991442 \\ \mathrm{~S} & -0.043129 & -1.814243 \\ \mathrm{C} & 3.277439 & -2.850263 \\ \mathrm{C} & 2.951837 & -2.910673 \\ \mathrm{C} & 1.716280 & -3.605583 \\ \mathrm{H} & 1.477982 & -4.477052 \\ \mathrm{H} & 1.794346 & -3.949613 \\ \mathrm{C} & 3.788151 & -2.269866 \\ \mathrm{C} & 4.894783 & -1.565533 \\ \mathrm{H} & 5.542030 & -1.059121 \\ \mathrm{C} & 5.189229 & -1.497975 \\ \mathrm{C} & 4.383813 & -2.145686 \\ \mathrm{C} & 4.673787 & -2.075839 \\ \mathrm{H} & 4.851593 & -3.075413 \\ \mathrm{H} & 5.561685 & -1.460704 \\ \mathrm{P} & -1.940210 & 0.655490 \\ \mathrm{C} & -3.061016 & 2.010202 \\ \mathrm{O} & 0.998033 & 0.010090 \\ \mathrm{H} & 2.632333 & -3.363281 \\ \mathrm{H} & -2.828835 & 2.139504 \\ \mathrm{H} & -6.101043 & 1.213402 \\ \mathrm{H} & 0.842553 & -2.929007 \\ \mathrm{H} & 3.554740 & -2.313137 \\ \mathrm{H} & 6.066703 & -0.936638 \\ \mathrm{H} & 3.825676 & -1.642949 \\ \mathrm{H} & -3.644778 & 2.292004 \\ & & \end{array}$

0.419373

$-1.691530$

$-1.458838$

$-1.574651$

$-1.867568$

$-2.801090$

$-1.379300$

$-1.083513$

$-0.936514$

1.578087

0.392661

$-0.956257$

$-1.409050$

$-0.778440$

$-2.452409$

$-1.865544$

$-1.426374$

$-2.151042$

$-0.073979$

0.855049

2.313736

2.745042

2.526314

2.405735

2.024757

$-0.784613$

1.119007

$-1.073396$

$-1.466304$

$-1.354001$

$-2.936422$

0.270328

2.873064

2.916106

$\mathrm{HAr}, \mathrm{Ar}=2,6-\mathrm{Me}_{2} \mathrm{C}_{6} \mathrm{H}_{3}$

\section{Enthalpy -310.653597 \\ Free energy -310.69539}

$\begin{array}{rrrr}\text { C } & 0.003081 & -0.944153 & -0.007295 \\ \text { C } & -1.214790 & -0.275223 & -0.003967 \\ \text { C } & -2.503785 & -1.024881 & 0.003458 \\ \text { H } & -2.349956 & -2.104393 & -0.152571 \\ \text { H } & -3.190246 & -0.667015 & -0.782420 \\ \text { C } & -1.204176 & 1.119644 & 0.000324 \\ \text { C } & -0.005760 & 1.810884 & 0.002158 \\ \text { H } & -0.008270 & 2.907618 & 0.000845 \\ \text { C } & 1.200801 & 1.124862 & -0.000391\end{array}$




$\begin{array}{rrrr}\mathrm{C} & 1.220821 & -0.265152 & -0.004453 \\ \mathrm{C} & 2.507022 & -1.019754 & 0.003853 \\ \mathrm{H} & 2.527873 & -1.802385 & -0.773164 \\ \mathrm{H} & 3.369986 & -0.356396 & -0.164663 \\ \mathrm{H} & 0.006015 & -2.043868 & -0.015072 \\ \mathrm{H} & -3.038518 & -0.904377 & 0.962098 \\ \mathrm{H} & -2.155224 & 1.667760 & -0.001637 \\ \mathrm{H} & 2.148021 & 1.679159 & -0.003035 \\ \mathrm{H} & 2.671031 & -1.533468 & 0.967497\end{array}$

$\mathrm{MoH}(\mathrm{S})\left(\mathrm{PMe}_{3}\right)(\mathrm{OMe})(\mathrm{SAr})_{2}$

\section{Enthalpy -2459.497059}

Free energy -2459.597926

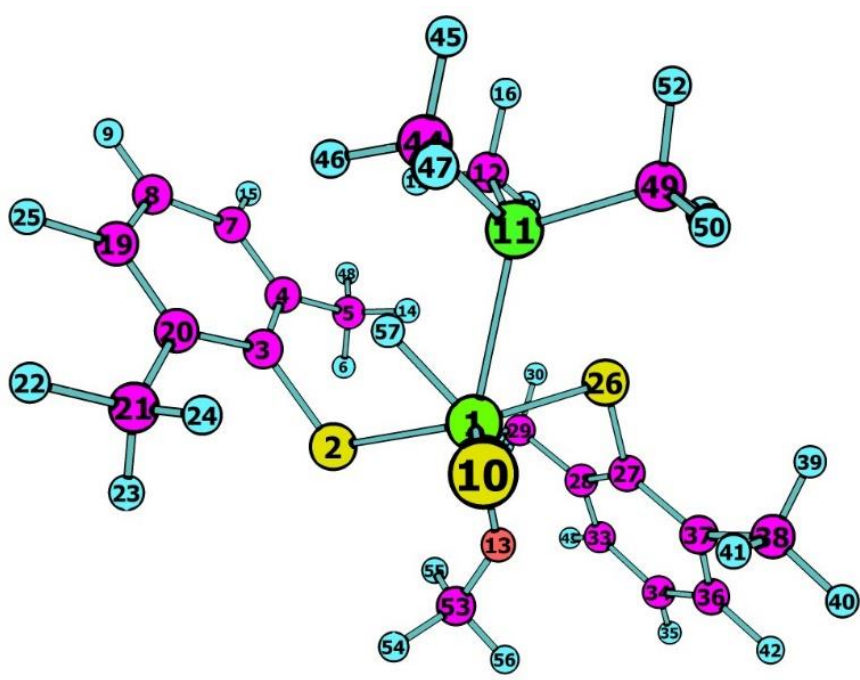

$\begin{array}{lr}\text { Mo } & 0.069964 \\ \mathrm{~S} & -1.067181 \\ \mathrm{C} & -2.693357 \\ \mathrm{C} & -2.918501 \\ \mathrm{C} & -1.818742 \\ \mathrm{H} & -1.305071 \\ \mathrm{C} & -4.204826 \\ \mathrm{C} & -5.243881 \\ \mathrm{H} & -6.250376 \\ \mathrm{~S} & -0.201530 \\ \mathrm{P} & -1.006419 \\ \mathrm{C} & -1.498467 \\ \mathrm{O} & 1.486427 \\ \mathrm{H} & -1.036640 \\ \mathrm{H} & -4.386415 \\ \mathrm{H} & -1.948085 \\ \mathrm{H} & -2.246420 \\ \mathrm{H} & -0.636709 \\ \mathrm{C} & -5.009095 \\ \mathrm{C} & -3.741119 \\ \mathrm{C} & -3.517285\end{array}$

0.539936

$-1.516496$

$-1.440989$

$-1.956698$

$-2.533437$

$-3.363808$

$-1.889573$

$-1.332704$

$-1.290907$

2.120131

2.084855

1.242684

$-0.474656$

$-1.785357$

$-2.288264$

1.957090

0.465484

0.764035

$-0.835196$

$-0.882977$

$-0.325766$
0.611381

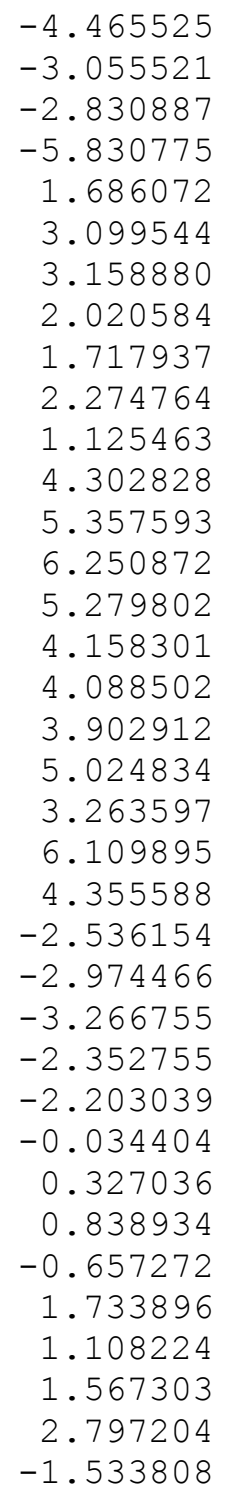

1.037148

0.351305

$-0.935034$

$-1.753831$

$-1.241461$

$-1.458476$

$-0.735750$

$-1.164684$

2.036978

$-1.109725$

$-2.620779$

1. 437281

$-1.978106$

$-2.463561$

$-3.329444$

$-2.392961$

$-3.110204$

0.532435

1.101115

2.459824
0.005940

$-1.060800$

0.540768

$-0.398584$

0.676202

$-0.262178$

$-1.633018$

$-2.353674$

$-1.909481$

$-3.409638$

$-2.326419$

$-2.333882$

$-1.703003$

$-2.271451$

$-0.351093$

0.392124

1.834361

2.472734

2.169261

2.038437

0.153495

$-3.406974$

2.844903

3.464630

2.070874

3.483303

$-2.912626$

3.498222

4.074157

3.181747

4.159405

$-1.627622$

$-1.739137$

$-2.531399$

$-1.644831$

0.605111
2. 908669

3.139356

2.442653

1. 112264

$-1.170209$

$-0.694749$

$-0.978850$

$-1.605208$

$-2.568298$

$-1.783424$

$-0.957702$

$-0.615421$

0.017229

0.296394

0.297573

$-0.049652$

0.301515

$-0.578226$

0.773050

1.007433

0.806022

$-0.834235$

$-0.543608$

$-1.342277$

$-0.256462$

0.333783

$-2.712859$

$-1.664185$

$-0.798160$

$-2.251365$

$-2.289076$

2. 109390

3. 021492

1. 484447

2. 422478

0.222362 
MoH..S TS

Enthalpy -2459.482842

Free energy -2459.58393

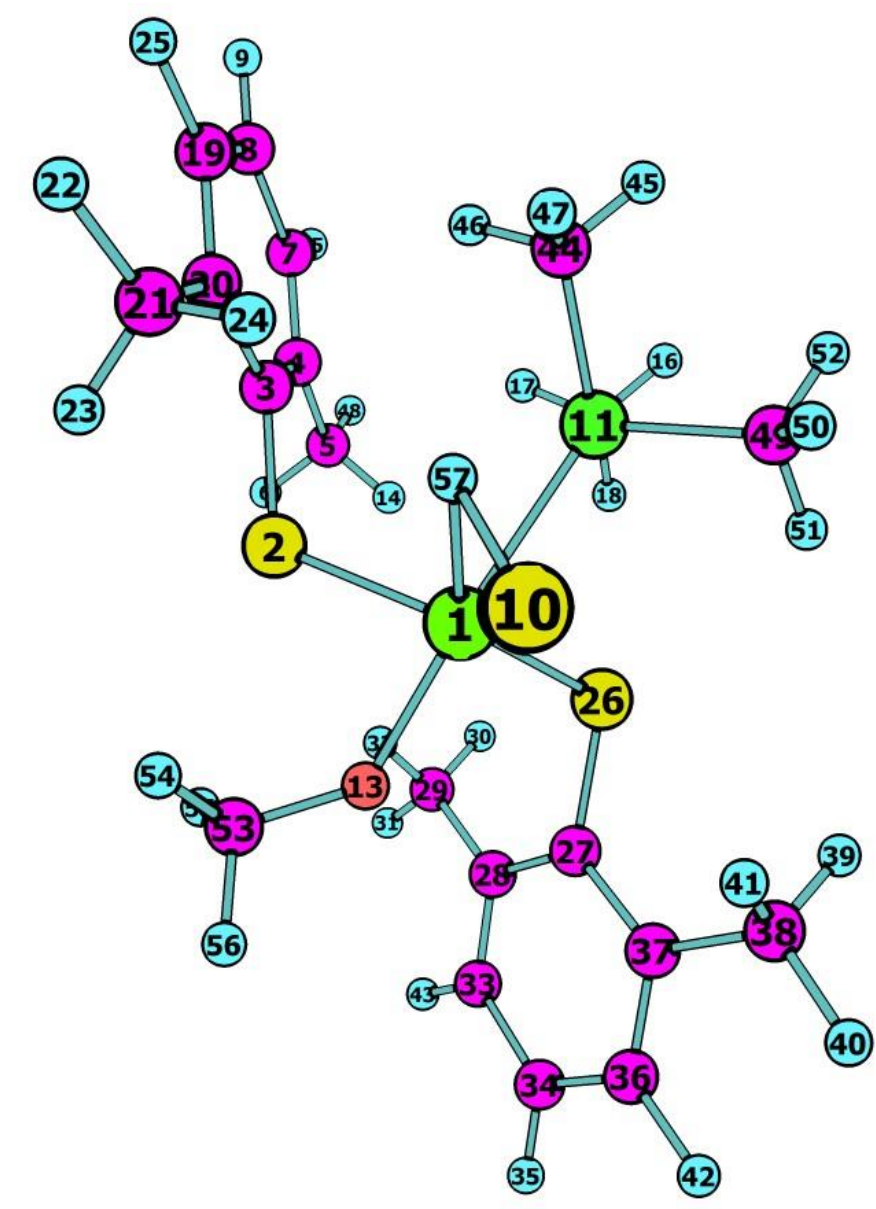

$\begin{array}{lr}\text { Mo } & 0.251911 \\ \mathrm{~S} & -1.199814 \\ \mathrm{C} & -2.872254 \\ \mathrm{C} & -3.318505 \\ \mathrm{C} & -2.425281 \\ \mathrm{H} & -2.163313 \\ \mathrm{C} & -4.643907 \\ \mathrm{C} & -5.519068 \\ \mathrm{H} & -6.562200 \\ \mathrm{~S} & 0.201811 \\ \mathrm{P} & -1.173941 \\ \mathrm{C} & -1.388467 \\ \mathrm{O} & 1.464742 \\ \mathrm{H} & -1.464994 \\ \mathrm{H} & -4.995799 \\ \mathrm{H} & -1.751172 \\ \mathrm{H} & -2.137569 \\ \mathrm{H} & -0.448538 \\ \mathrm{C} & -5.078821 \\ \mathrm{C} & -3.757445\end{array}$
0.341444
0.607895
$-1.506935$
0.694301
0.262003
$-1.585575$
$-1.004877$
$-2.302341$
$-1.956188$
$-3.306044$
$-1.579167$
$-1.348665$
$-1.348424$
$-0.756765$
$-0.455175$
$-0.585813$
$-0.740177$
1.963523
2.131291
1.712547
1.108481
$-1.064383$
$-2.751783$
$-0.968705$
1.320184
$-1.786317$
$-2.122166$
$-1.658704$
$-2.339446$
1. 925070
$-3.397738$
0.303418
$-2.778310$
0.722802
$-3.173839$
$-0.404572$
0.806522
$-0.602894$
1.192610

$\begin{array}{rrr}-3.329460 & -0.200253 & 2.557476 \\ -4.202672 & -0.024095 & 3.203400 \\ -2.691611 & -0.958200 & 3.039253 \\ -2.741062 & 0.735480 & 2.553275 \\ -5.775724 & 0.041585 & 1.525752 \\ 1.714007 & 0.592731 & -1.209842 \\ 3.238540 & -0.149311 & -0.714824 \\ 3.440923 & -1.520209 & -0.928266 \\ 2.380903 & -2.385753 & -1.506749 \\ 2.049475 & -2.034138 & -2.498485 \\ 2.736325 & -3.421302 & -1.618641 \\ 1.479133 & -2.402234 & -0.868676 \\ 4.664205 & -2.068709 & -0.566535 \\ 5.661916 & -1.288582 & -0.009541 \\ 6.621286 & -1.739288 & 0.265276 \\ 5.446351 & 0.061008 & 0.193694 \\ 4.236897 & 0.655217 & -0.149842 \\ 4.030777 & 2.107575 & 0.095238 \\ 3.761420 & 2.652506 & -0.824977 \\ 4.941592 & 2.568222 & 0.506743 \\ 3.209251 & 2.292618 & 0.810481 \\ 6.234087 & 0.683695 & 0.633892 \\ 4.832251 & -3.139972 & -0.729763 \\ -2.856966 & 2.198952 & -0.629297 \\ -3.216807 & 2.975236 & -1.325149 \\ -3.546286 & 1.344401 & -0.685563 \\ -2.898995 & 2.610597 & 0.392160 \\ -2.917527 & -2.434229 & -2.931795 \\ -0.395645 & 3.326498 & -1.311471 \\ -0.344450 & 3.874880 & -0.358017 \\ 0.630554 & 3.222541 & -1.694448 \\ -0.978743 & 3.929123 & -2.027953 \\ 1.352040 & -1.986630 & 2.220209 \\ 0.538090 & -1.841034 & 2.961883 \\ 1.180355 & -2.958926 & 1.711908 \\ 2.307341 & -2.078801 & 2.772749 \\ -1.029232 & 1.294603 & 1.369542\end{array}$


$\mathrm{Mo}(\mathrm{SH})\left(\mathrm{PMe}_{3}\right)(\mathrm{OMe})(\mathrm{SAr})_{2}$

\section{Enthalpy -2459.497059}

Free energy -2459.597926

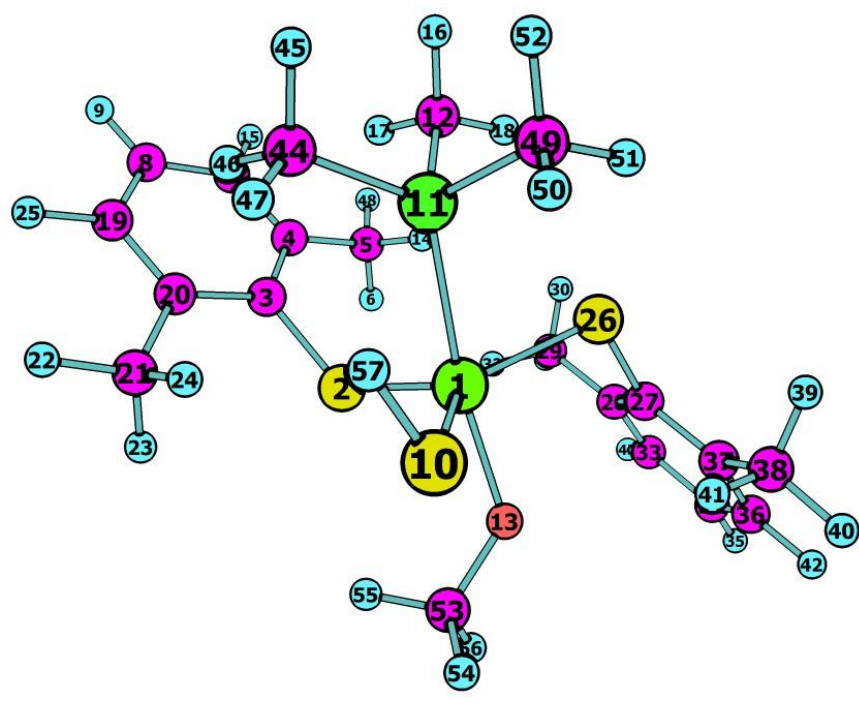

$\begin{array}{lr}\text { Mo } & 0.246552 \\ \text { S } & -1.024467 \\ \mathrm{C} & -2.642184 \\ \mathrm{C} & -2.837134 \\ \mathrm{C} & -1.704377 \\ \mathrm{H} & -1.169268 \\ \mathrm{C} & -4.130111 \\ \mathrm{C} & -5.200537 \\ \mathrm{H} & -6.211920 \\ \mathrm{~S} & 0.007312 \\ \mathrm{P} & -1.307151 \\ \mathrm{C} & -1.687257 \\ \mathrm{O} & 1.639175 \\ \mathrm{H} & -0.950531 \\ \mathrm{H} & -4.291829 \\ \mathrm{H} & -2.238069 \\ \mathrm{H} & -2.318659 \\ \mathrm{H} & -0.769065 \\ \mathrm{C} & -4.992756 \\ \mathrm{C} & -3.718548 \\ \mathrm{C} & -3.526857 \\ \mathrm{H} & -4.489401 \\ \mathrm{H} & -3.064889 \\ \mathrm{H} & -2.855591 \\ \mathrm{H} & -5.840028 \\ \mathrm{~S} & 1.650075 \\ \mathrm{C} & 3.106471 \\ \mathrm{C} & 3.157920 \\ \mathrm{C} & 1.986378 \\ \mathrm{H} & 1.600265 \\ \mathrm{H} & 2.245923 \\ \mathrm{H} & 1.146174 \\ & \end{array}$
$\mathrm{C}$
$\mathrm{C}$
$\mathrm{H}$
$\mathrm{C}$
$\mathrm{C}$
$\mathrm{C}$
$\mathrm{H}$
$\mathrm{H}$
$\mathrm{H}$
$\mathrm{H}$
$\mathrm{H}$
$\mathrm{C}$
$\mathrm{H}$
$\mathrm{H}$
$\mathrm{H}$
$\mathrm{H}$
$\mathrm{C}$
$\mathrm{H}$
$\mathrm{H}$
$\mathrm{H}$
$\mathrm{C}$
$\mathrm{H}$

$\begin{array}{rrr}-0.700764 & -0.339472 & \mathrm{H} \\ 0.901510 & -1.382032 & \mathrm{H} \\ 1.230148 & -0.740411 & \mathrm{H}\end{array}$

0.141580

$3.133562 \quad 0.626969$

$3.628138-0.201163$

$2.565884 \quad 0.579673$

$1.805290 \quad 0.147097$

$2.030717 \quad 0.500396$

$-3.005062-0.946448$

$-1.359765 \quad 1.526793$

$-0.015094 \quad 2.676842$

$-0.847347-1.657755$

2.5299391 .162820

3.397034

$-0.408718$

0.747361

0.471383

0.765701

0.459796

$-0.668559$

$-1.144290$

$-0.341076$

$-1.445080$

0.166473

0.004461

0.721440

2.091759

2.981391

2. 926301

4.030209

2. 707998
2.615308

1.809967

2. 240108

0.459377

$-0.109957$

$-1.567568$

$-1.776973$

$-2.041839$

$-2.074541$

$-0.183614$

3. 687113

$-2.046863$

$-2.339078$

$-1.299489$

$-2.940726$

3. 915840

$-2.592971$

$-3.543962$

$-2.243160$

$-2.793429$

$-1.080783$

$-2.139654$

$-0.845685$

$-0.466223$

$-3.422345$
$-0.195019$

$-0.418932$

$-0.821880$

$-0.129444$

0.385076

0.674760

1.733298

0.440374

0.081649

$-0.306047$

$-0.421240$

1. 248981

2.209786

0.775265

0.605913

1. 314732

2.633773

2.107268

3.014696

3. 494737

$-2.998522$

$-3.241108$

$-3.376899$

$-3.555609$

$-0.330866$
HSAr, $\mathrm{Ar}=2,6-\mathrm{Me}_{2} \mathrm{C}_{6} \mathrm{H}_{3}$

\section{Enthalpy -708.800777 \\ Free energy -708.845094}

$\begin{array}{rrrr}\mathrm{S} & -2.087607 & 0.400223 & -0.004067 \\ \mathrm{C} & -0.351091 & 0.093603 & 0.003638 \\ \mathrm{C} & 0.026493 & -1.257199 & 0.001181 \\ \mathrm{C} & -1.001354 & -2.332986 & -0.003691 \\ \mathrm{H} & -1.651193 & -2.286298 & -0.897102 \\ \mathrm{C} & 1.380431 & -1.567670 & 0.000815 \\ \mathrm{C} & 2.341852 & -0.572274 & 0.004082 \\ \mathrm{H} & 3.406150 & -0.831586 & 0.005349 \\ \mathrm{H} & -1.671411 & -2.273440 & 0.873516 \\ \mathrm{H} & 1.677707 & -2.623910 & -0.000463 \\ \mathrm{C} & 1.949059 & 0.754304 & 0.003038 \\ \mathrm{C} & 0.605781 & 1.119327 & -0.000567 \\ \mathrm{C} & 0.230085 & 2.559614 & -0.011084 \\ \mathrm{H} & 1.126452 & 3.197916 & -0.038644 \\ \mathrm{H} & -0.386102 & 2.828409 & -0.888758 \\ \mathrm{H} & -0.344896 & 2.855189 & 0.886208 \\ \mathrm{H} & 2.705605 & 1.549212 & 0.001567 \\ \mathrm{H} & -0.534029 & -3.329584 & 0.006812 \\ \mathrm{H} & -2.014091 & 1.730211 & 0.132125\end{array}$




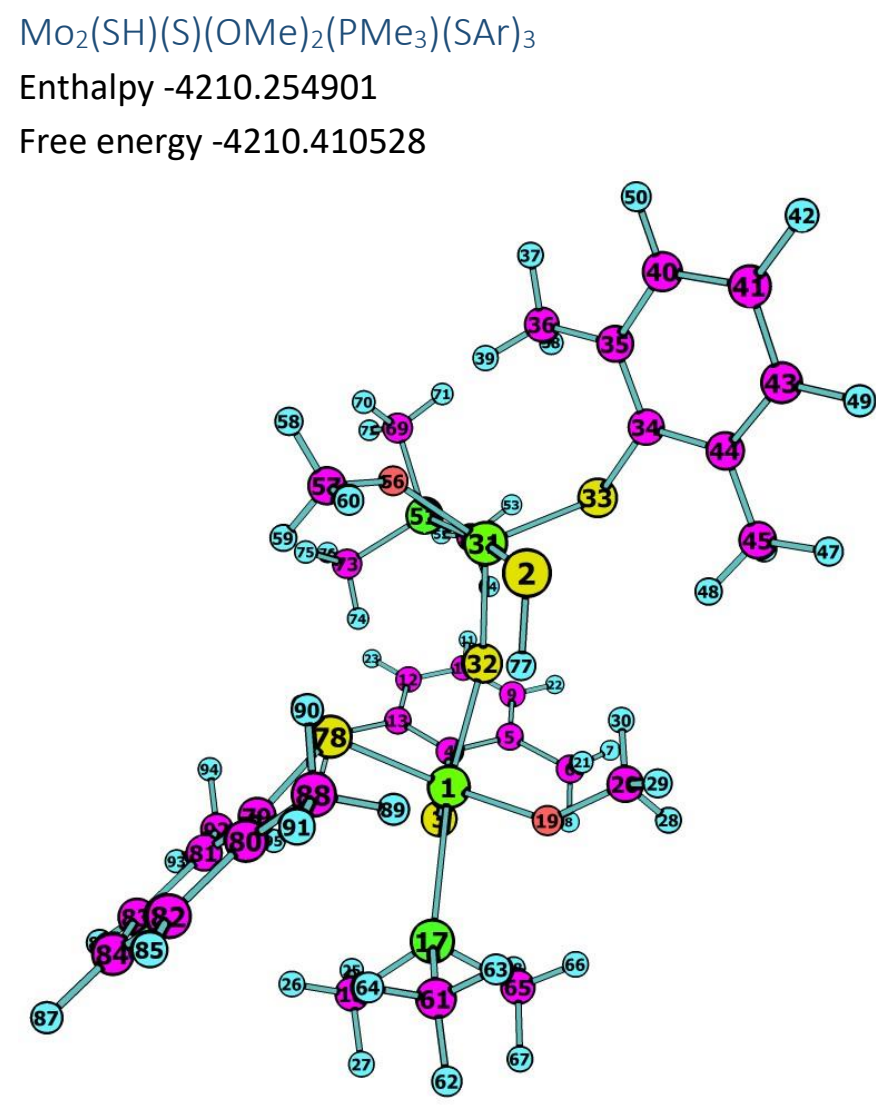

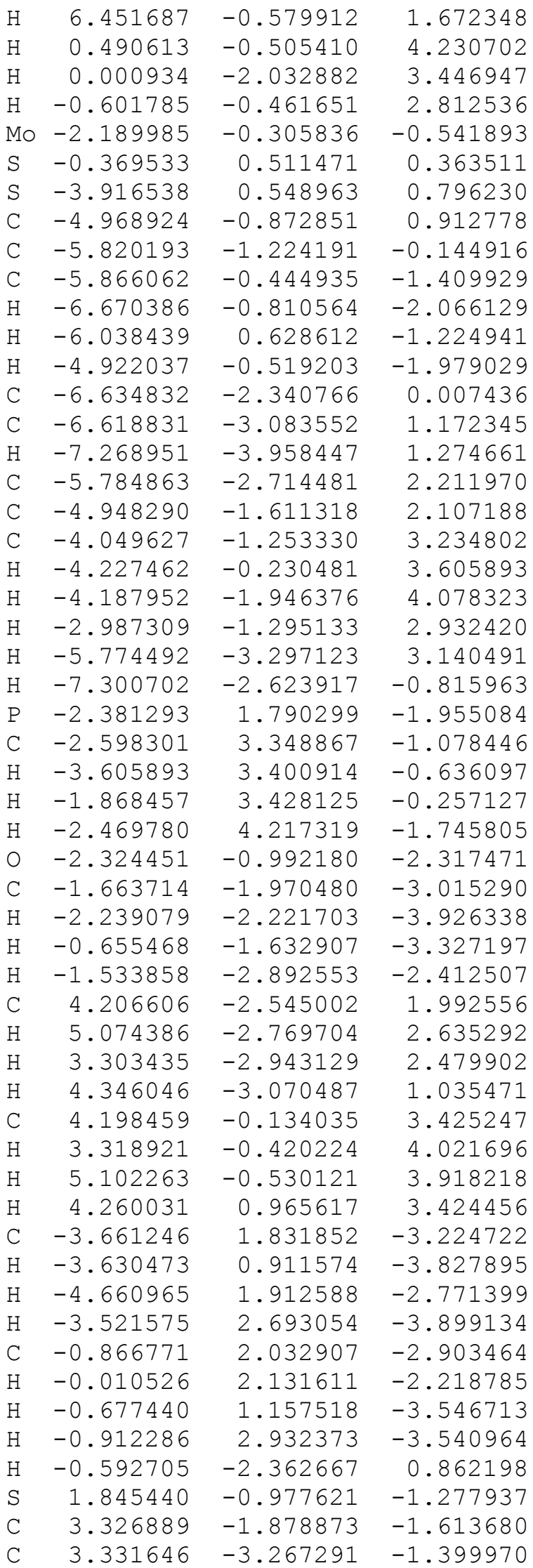

$\begin{array}{lrrr}\text { Mo } & 1.791587 & 0.031621 & 0.835062 \\ \mathrm{~S} & -1.814973 & -2.512265 & 0.306305 \\ \mathrm{~S} & 2.720634 & 2.149130 & 1.031776 \\ \mathrm{C} & 1.583811 & 3.443999 & 0.625052 \\ \mathrm{C} & 0.750946 & 3.966323 & 1.625403 \\ \mathrm{C} & 0.721748 & 3.392158 & 2.997119 \\ \mathrm{H} & 0.001910 & 3.929307 & 3.633032 \\ \mathrm{H} & 1.708160 & 3.437222 & 3.489104 \\ \mathrm{C} & -0.067601 & 5.043408 & 1.304274 \\ \mathrm{C} & -0.070288 & 5.586708 & 0.032200 \\ \mathrm{H} & -0.722428 & 6.435277 & -0.200200 \\ \mathrm{C} & 0.740279 & 5.045372 & -0.948335 \\ \mathrm{C} & 1.586140 & 3.976492 & -0.671991 \\ \mathrm{C} & 2.454810 & 3.418778 & -1.742634 \\ \mathrm{H} & 3.527261 & 3.525615 & -1.504963 \\ \mathrm{H} & 2.271878 & 3.927712 & -2.701523 \\ \mathrm{P} & 4.033365 & -0.766920 & 1.740019 \\ \mathrm{C} & 5.617116 & -0.294217 & 1.009892 \\ \mathrm{O} & 1.385033 & -0.916462 & 2.446476 \\ \mathrm{C} & 0.278111 & -0.978210 & 3.250416 \\ \mathrm{H} & 0.435013 & 2.324528 & 2.990603 \\ \mathrm{H} & -0.718432 & 5.461906 & 2.081159 \\ \mathrm{H} & 0.732418 & 5.464281 & -1.962159 \\ \mathrm{H} & 2.283459 & 2.338145 & -1.892618 \\ \mathrm{H} & 5.665595 & 0.795355 & 0.853970 \\ \mathrm{H} & 5.772118 & -0.785981 & 0.037719\end{array}$




$\begin{array}{lrrr}\mathrm{C} & 4.433557 & -1.236321 & -2.190263 \\ \mathrm{C} & 4.469749 & -3.988898 & -1.741026 \\ \mathrm{C} & 5.548097 & -2.000573 & -2.519130 \\ \mathrm{C} & 5.572208 & -3.364717 & -2.295650 \\ \mathrm{H} & 4.481214 & -5.071445 & -1.566486 \\ \mathrm{H} & 6.416962 & -1.501878 & -2.965492 \\ \mathrm{H} & 6.458553 & -3.949571 & -2.562500 \\ \mathrm{C} & 2.163786 & -3.966556 & -0.800388 \\ \mathrm{H} & 1.911310 & -3.570016 & 0.199860 \\ \mathrm{H} & 1.249812 & -3.845772 & -1.407370 \\ \mathrm{H} & 2.361714 & -5.044311 & -0.696733 \\ \mathrm{C} & 4.446073 & 0.230379 & -2.432958 \\ \mathrm{H} & 5.403854 & 0.546851 & -2.873557 \\ \mathrm{H} & 3.638994 & 0.542543 & -3.117706 \\ \mathrm{H} & 4.293921 & 0.806307 & -1.502326\end{array}$

$\mathrm{MO}_{2}$

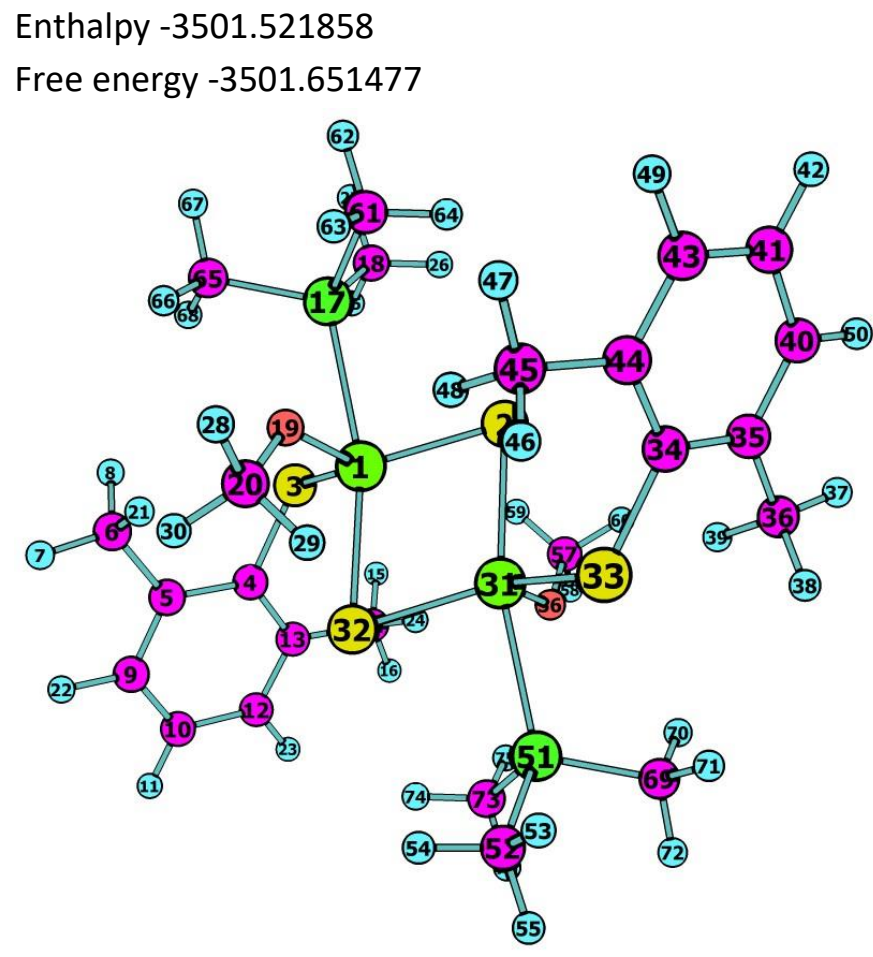

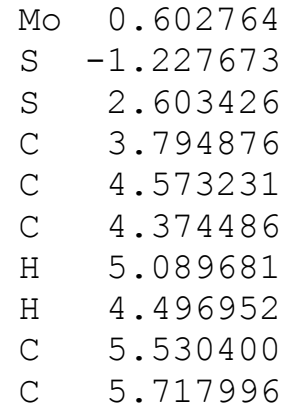

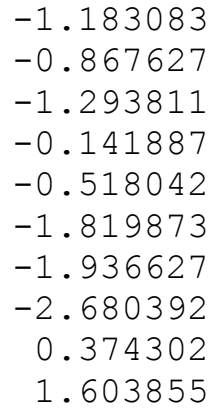

0.092826

$-1.160935$

$-1.132134$

$-0.507444$

0.596268

1.288416

2.116904

0.609108

1.065058

0.461535

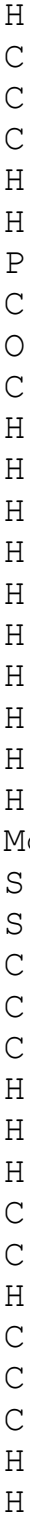

c

3.107594

3.103507

3.439597

0.329761

0.251685

0.432813

0.471688

3.355650

6.141098

5.079793

2.057700

1.147605

$-0.624826$

0.185000

0.014688

$-0.082721$

1.512030

Mo -0.602720

1. 227720

$-2.603354$

$-3.794891$

$-4.573249$

$-4.374427$

$-5.089664$

$-4.496763$

$-3.355611$

$-5.530502$

$-5.718182$

$-6.479827$

$-4.939797$

$-3.969443$

$-3.107718$

$-3.103620$

$-3.439749$

$-2.057828$

$-5.080058$

$-6.141200$

$-0.329704$

$-0.251503$

$-1.147369$

0.625062

$-0.184847$

$-0.432831$

$-0.471796$

$-0.014926$

0.082695

$-1.512154$

$-1.160453$

$-1.220849$

$-1.167070$

$-2.058827$
2.292227

1.960627

1.101860

1.540510

0.821007

2.514801

$-3.601506$

$-4.045142$

$-2.096787$

$-1.638502$

$-1.909302$

0.087760

2.936224

1.648228

$-3.682996$

$-3.576745$

$-5.136196$

$-2.397198$

$-0.688915$

$-1.473799$

1.183035

0.867585

1.293758

0.141932

0.518175

1.820007

1.936851

2.680524

1.909347

$-0.374083$

$-1.603631$

$-2.291932$

$-1.960486$

$-1.101809$

$-1.540539$

$-0.821078$

$-2.514841$

$-1.648263$

$-2.936078$

$-0.087473$

3.601463

4.045101

3.682925

3.576737

5.136158

2.096744

1.638426

2.397144

0.688891

1.473609

$-4.325987$

$-5.409680$

$-4.165904$

$-3.845311$
0.842330

$-0.624076$

$-1.128083$

$-2.256099$

$-3.091699$

$-2.647209$

$-0.572111$

$-2.315426$

1.765759

3.061991

1.709488

1.929541

$-1.105047$

$-1.931447$

$-2.844926$

$-2.789632$

$-2.459728$

3.726864

3.198098

3.406911

$-0.092837$

1.160919

1.132173

0.507462

$-0.596219$

$-1.288344$

$-2.116784$

$-0.609010$

$-1.709481$

$-1.065004$

$-0.461500$

$-0.842283$

0.624088

1.128086

2.256086

3.091723

2. 647144

1.931431

1.105048

$-1.929464$

0.572082

2.315390

2.844962

2.789527

2.459685

$-1.765779$

$-3.061996$

$-3.726933$

$-3.198131$

$-3.406815$

0.136485

$-0.059342$

1.225875

$-0.283177$ 


$\begin{array}{lrrr}\mathrm{C} & 1.652736 & -4.637596 & 0.080710 \\ \mathrm{H} & 1.782971 & -4.449703 & 1.157719 \\ \mathrm{H} & 1.438419 & -5.709806 & -0.062729 \\ \mathrm{H} & 2.604942 & -4.406984 & -0.421798 \\ \mathrm{C} & -1.652753 & 4.637525 & -0.080635 \\ \mathrm{H} & -1.783139 & 4.449555 & -1.157612 \\ \mathrm{H} & -2.604889 & 4.406964 & 0.422027 \\ \mathrm{H} & -1.438402 & 5.709744 & 0.062693 \\ \mathrm{C} & 1.160447 & 4.325963 & -0.136628 \\ \mathrm{H} & 2.058857 & 3.845268 & 0.282935 \\ \mathrm{H} & 1.166965 & 4.165916 & -1.226024 \\ \mathrm{H} & 1.220868 & 5.409650 & 0.059228\end{array}$

trans- $\mathrm{MoH}\left(\mathrm{PMe}_{3}\right)(\mathrm{SAr})_{3}, \mathrm{Ar}=2,6-\mathrm{Me}_{2} \mathrm{C}_{6} \mathrm{H}_{3}$, isomer 1.

\section{Enthalpy -2654.474901}

Free energy -2654.581709

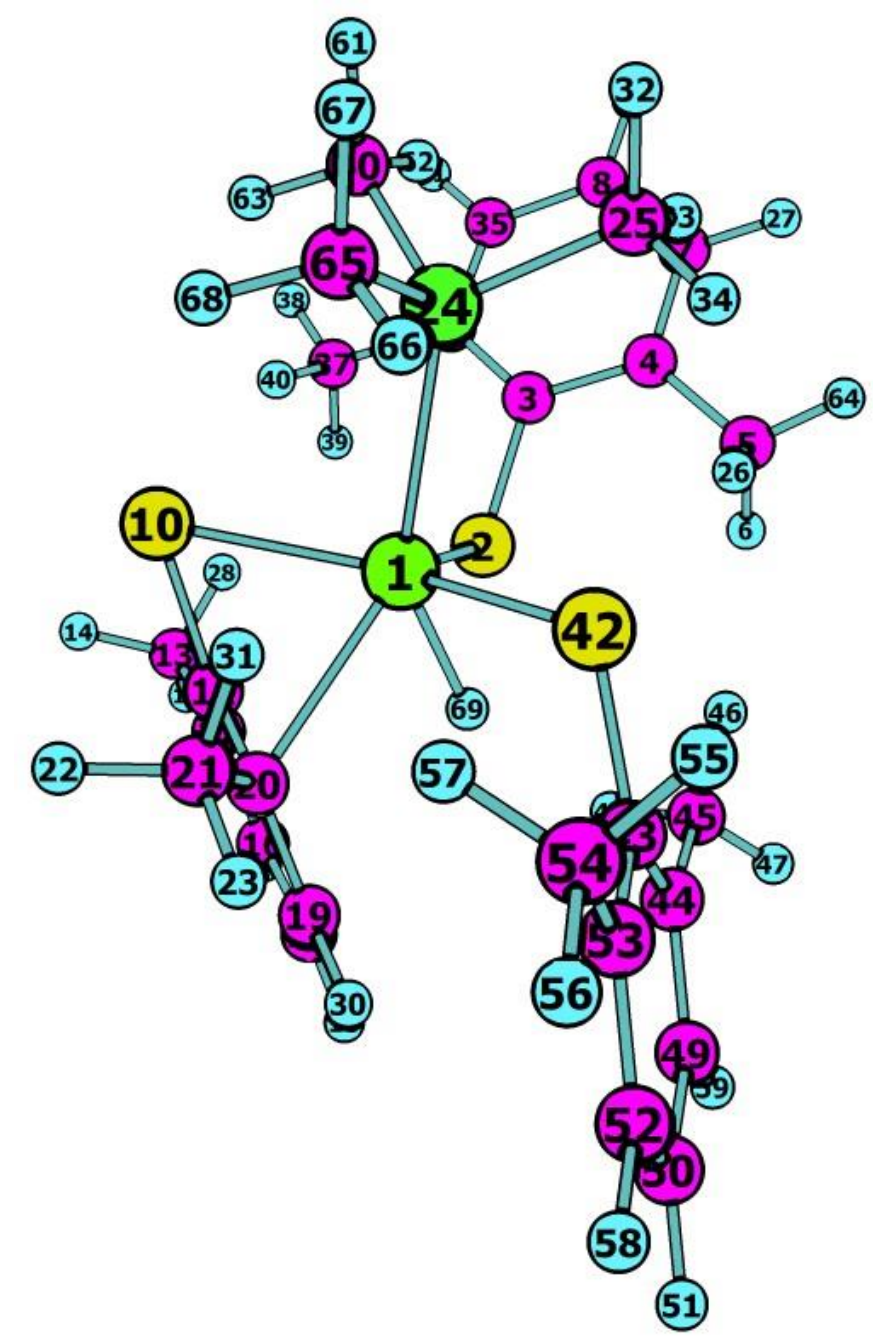

$\begin{array}{lrrr}\text { Mo } & -0.028475 & 0.298453 & 0.448910 \\ \text { S } & 1.310934 & 0.520902 & -1.442041\end{array}$

\begin{tabular}{|c|c|c|}
\hline 1207 & -0.223982 & -1.302866 \\
\hline 3.077206 & -1.562388 & -1.697868 \\
\hline 28759 & -2.388983 & -2.1561 \\
\hline 1.472666 & -1.977067 & -3.0728 \\
\hline 243 & -2.115938 & \\
\hline 7942 & -1.363293 & \\
\hline 45758 & -1.813895 & -1 \\
\hline 35009 & 817 & \\
\hline 891 & 2280 & \\
\hline 497 & & \\
\hline 7497 & 2 & \\
\hline 0 . & & \\
\hline 0 . & & \\
\hline 1. & & \\
\hline 2 . & & \\
\hline-3 & & \\
\hline-2 & 1. & -0 \\
\hline-1 & & \\
\hline-2 & & \\
\hline-2 . & & \\
\hline-3 & & \\
\hline 868 & -1 & .8 \\
\hline 1.9 & -2 . & 1.4 \\
\hline 1.1 & -2 . & -1 \\
\hline 4.4 & -3 & -1 \\
\hline 1.5 & 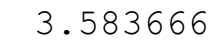 & -0 \\
\hline-1.4 & 4. & -2 \\
\hline-3.9 & 1. & -0 \\
\hline-1.729 & & ? \\
\hline 2.42372 & -3 & .2 \\
\hline 2.5 & -2 . & .5 \\
\hline 0.9 & -3 & .2 \\
\hline 5.2 & -0 & -0 \\
\hline 4. & & -0 \\
\hline 3.8 & & -0 \\
\hline $4 . \varepsilon$ & & -0 \\
\hline 3.5 & & -1 \\
\hline 3.1 & & \\
\hline 6.1 & & -0 \\
\hline 1.2 & -1 & 0.3 \\
\hline $2 . \varepsilon$ & -1 & -0 \\
\hline-2. & -1 & $-1 \cdot 7$ \\
\hline-1.8 & -1 & -2 \\
\hline 9654 & -1 & -2 \\
\hline-2.123690 & -1 & \\
\hline-1.536 & -0 . & -2 \\
\hline-4.271769 & -1 & -2 \\
\hline-5.388168 & -1 & -1 \\
\hline-6.3 & -1 & -1 \\
\hline-5.2 & -1 & -0 . \\
\hline-3. & -1 & \\
\hline-3.865011 & -2 . & \\
\hline-3.373 & -3.205112 & \\
\hline-4.863190 & -2.31267 & 2.265 \\
\hline
\end{tabular}




$\begin{array}{rrrr}\mathrm{H} & -3.269660 & -1.539563 & 2.440103 \\ \mathrm{H} & -6.115869 & -1.714661 & 0.481417 \\ \mathrm{H} & -4.385474 & -0.837469 & -3.345455 \\ \mathrm{C} & 3.232359 & -0.510315 & 2.237131 \\ \mathrm{H} & 3.626094 & -1.063650 & 3.106740 \\ \mathrm{H} & 3.935366 & -0.645356 & 1.401919 \\ \mathrm{H} & 3.209525 & 0.560755 & 2.494525 \\ \mathrm{H} & 2.249217 & -3.419124 & -2.375550 \\ \mathrm{C} & 0.807164 & -1.236940 & 3.438561 \\ \mathrm{H} & -0.183572 & -1.713565 & 3.361830 \\ \mathrm{H} & 1.425841 & -1.839177 & 4.126566 \\ \mathrm{H} & 0.669163 & -0.239829 & 3.886906 \\ \mathrm{H} & -0.916703 & 0.447733 & -0.920002\end{array}$

trans- $\mathrm{MoH}\left(\mathrm{PMe}_{3}\right)(\mathrm{SAr})_{3}, \mathrm{Ar}=2,6-\mathrm{Me}_{2} \mathrm{C}_{6} \mathrm{H}_{3}$, isomer 2.

\section{Enthalpy -2654.477248}

\section{Free energy -2654.587618}

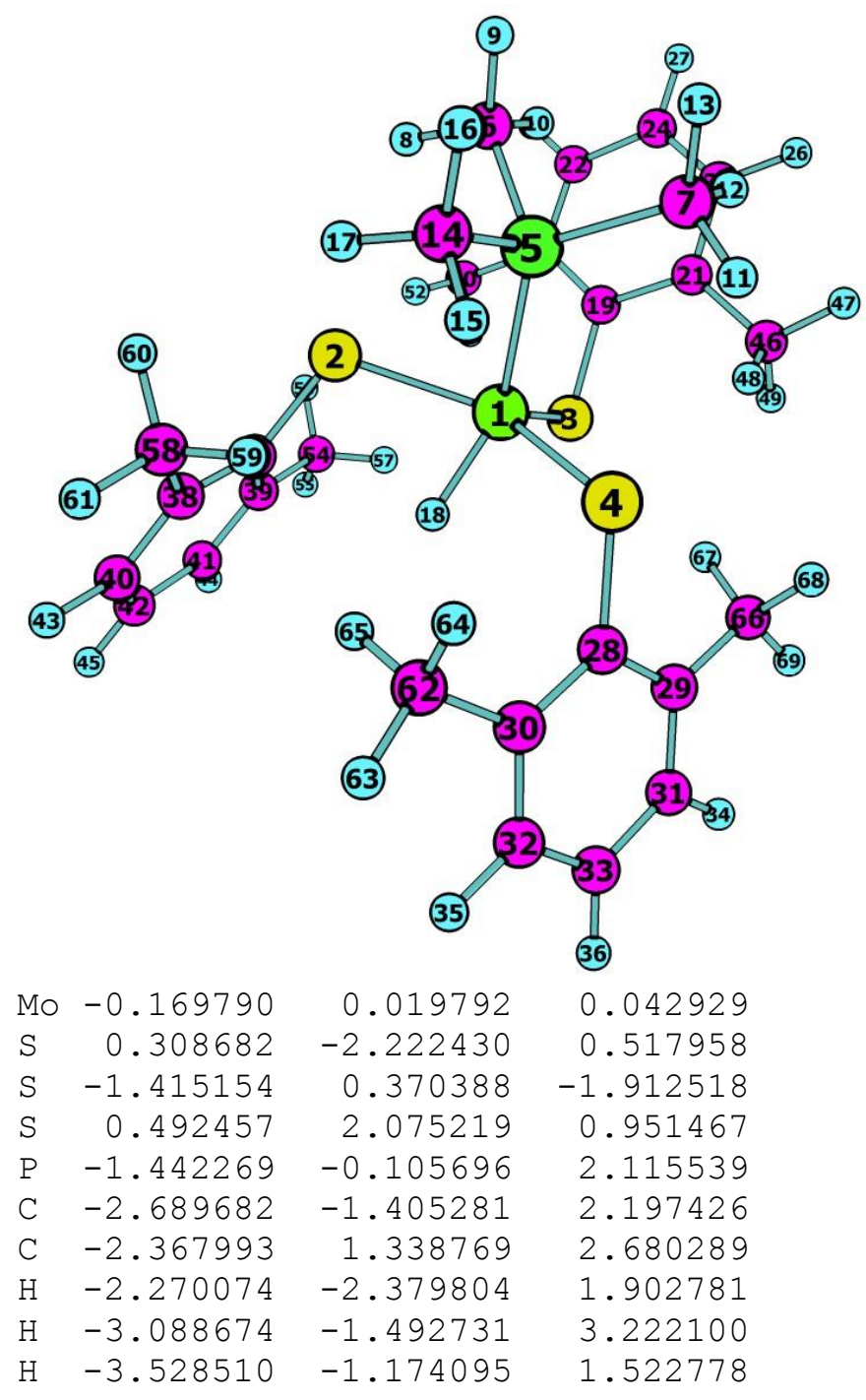

\begin{tabular}{|c|c|c|}
\hline-1.724796 & 2.229651 & \\
\hline-3.201704 & 1.560604 & \\
\hline-2.792090 & 1.147407 & \\
\hline-0.371141 & -0.457374 & \\
\hline 0.429043 & 0.295228 & 7 \\
\hline-0.955014 & -0.442951 & \\
\hline 0.09580 & -1 & \\
\hline & -0 . & \\
\hline-3 & & \\
\hline-3. & -1 & \\
\hline-3 & & \\
\hline-4.8 & -1 & \\
\hline-5 & 0 & \\
\hline-5 & -0 . & U. \\
\hline-5.2 & -2 & -0 . \\
\hline-5 . & 1. & \\
\hline-6. & -0 . & \\
\hline $1 . \varepsilon$ & 2 . & \\
\hline 1. & 3. & -1 . \\
\hline 3.7 & 2 . & \\
\hline 2. & 4. & -1 . \\
\hline 4.2 & 2 . & -0 . \\
\hline 4. & 3. & -1 \\
\hline 2. & 4. & -2 . \\
\hline 5.2 & 2. & 0 . \\
\hline 4.9 & 4.2 & -1 \\
\hline 1.8 & -2.6 & -0 . \\
\hline 2.9 & -2 & 0.7 \\
\hline 2.0 & -3 & $-1.4 \varepsilon$ \\
\hline 4.2 & -3. & 0 . \\
\hline 3.3 & -3 & -1 \\
\hline 4.7 & -3 & -1 \\
\hline 5. & -3 & 0 \\
\hline 3. & -3 & -2 \\
\hline 5.3 & -3 & -1 \\
\hline-3.2 & 2 . & -0 \\
\hline-3 & 3. & -0 \\
\hline-2 & 2 . & -0 \\
\hline-3 & 2 . & -1 \\
\hline & -2 . & -1 \\
\hline-2 & -2 . & -2 \\
\hline-1. & -2 . & -1 \\
\hline-3. & -3 & -1 \\
\hline 0. & -3 & \\
\hline 1.2 & -3 & \\
\hline 0.0 & & \\
\hline 0.4 & -2 . & -2 \\
\hline 2.8 & -2 . & \\
\hline 2.4 & -1 & \\
\hline 2.1 & -3 & \\
\hline 3.8 & -2 & 2.70 \\
\hline 3.4 & 1.5895 & \\
\hline 4.5 & 1.592126 & \\
\hline 2.8 & 1.919568 & \\
\hline
\end{tabular}




$\begin{array}{rr}\mathrm{H} & 3.160627 \\ \mathrm{C} & 0.303587 \\ \mathrm{H} & -0.277465 \\ \mathrm{H} & -0.283336 \\ \mathrm{H} & 0.346138\end{array}$

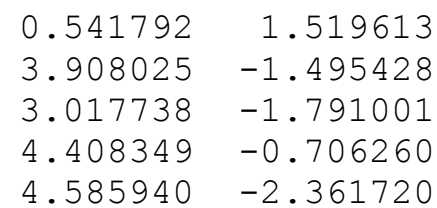

$\mathrm{MoH}\left(\mathrm{PMe}_{3}\right)\left(\mathrm{SMe}_{3}\right.$ (gas phase calculation)

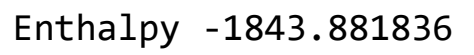

Free energy -1843.955261

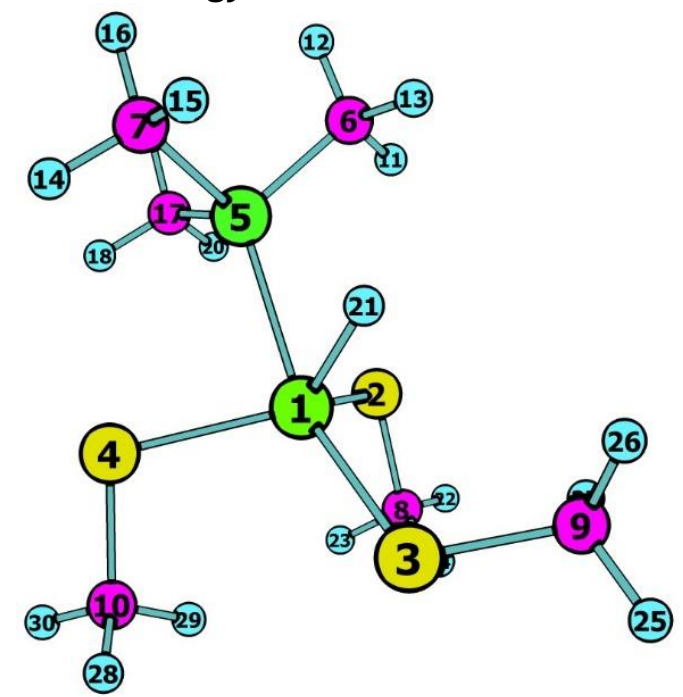

\begin{tabular}{lr} 
Mo & 0.294052 \\
$\mathrm{~S}$ & 0.484624 \\
$\mathrm{~S}$ & 2.241630 \\
$\mathrm{~S}$ & -0.271386 \\
$\mathrm{P}$ & -2.075462 \\
$\mathrm{C}$ & -2.559215 \\
$\mathrm{C}$ & -3.013332 \\
$\mathrm{C}$ & 2.113942 \\
$\mathrm{C}$ & 2.781894 \\
$\mathrm{C}$ & 1.290447 \\
$\mathrm{H}$ & -2.131344 \\
$\mathrm{H}$ & -3.654325 \\
$\mathrm{H}$ & -2.163785 \\
$\mathrm{H}$ & -2.892930 \\
$\mathrm{H}$ & -2.626989 \\
$\mathrm{H}$ & -4.088800 \\
$\mathrm{C}$ & -2.972292 \\
$\mathrm{H}$ & -2.874517 \\
$\mathrm{H}$ & -4.043474 \\
$\mathrm{H}$ & -2.539830 \\
$\mathrm{H}$ & -0.269214 \\
$\mathrm{H}$ & 2.347541 \\
$\mathrm{H}$ & 2.118820 \\
$\mathrm{H}$ & 2.897935 \\
$\mathrm{H}$ & 3.817832 \\
$\mathrm{H}$ & 2.148376 \\
$\mathrm{H}$ & 2.739515 \\
& \\
\hline
\end{tabular}

\begin{abstract}
0.171872
$-1.602896$

1.394814

$-0.188365$

0.247470

0.433128

1.558494

$-2.350542$

1.686382

$-0.690881$

$-0.387053$

0.426622

1.375640

1.478719

2.544174

1.515092

$-1.247707$

$-1.409189$

$-1.185115$

$-2.125736$

1.520739

$-3.005199$

$-2.968342$

$-1.580300$

2.058050

2.450965

0.767529
\end{abstract}

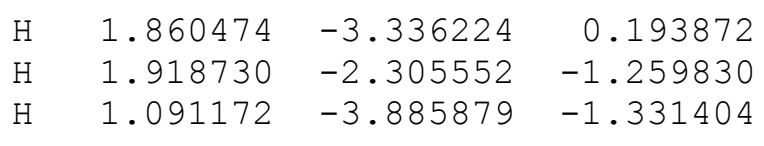

trans- $\mathrm{MoH}\left(\mathrm{PMe}_{3}\right)(\mathrm{SMe})_{3}$ (gas phase calculation)
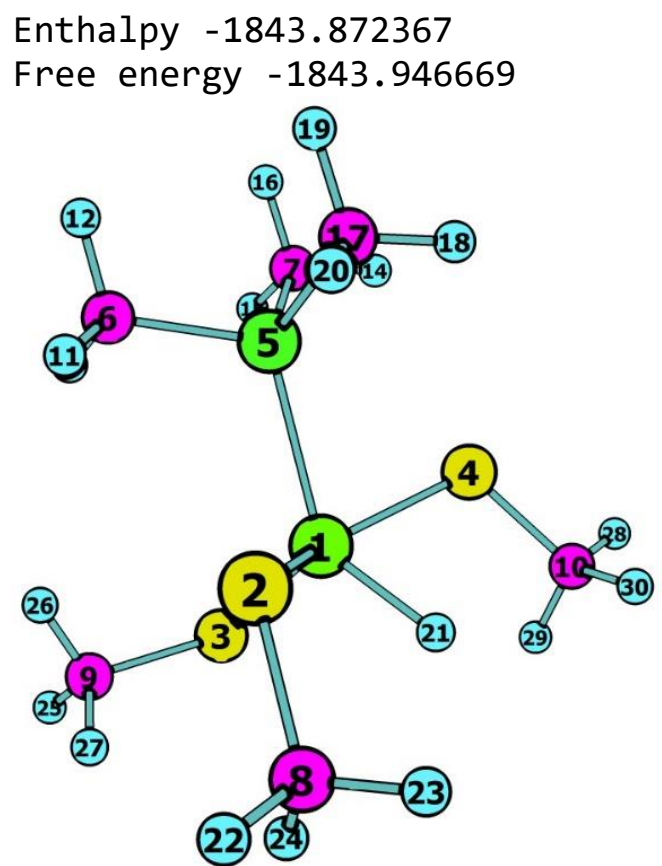


$\begin{array}{lrrr}\mathrm{H} & -1.077302 & -4.492118 & -0.282341 \\ \mathrm{H} & 0.567569 & -3.823513 & -0.039174 \\ \mathrm{H} & -0.247642 & -3.652956 & -1.623396\end{array}$

